\title{
Constraining the abundance of dark matter in the central region of the galaxy cluster MACS J1206.2-0847 with a free-form strong lensing analysis
}

\author{
Alberto Manjón-García ${ }^{1,2}$, Jose M. Diego ${ }^{1}$, Diego Herranz ${ }^{1,2}$, and Daniel Lam ${ }^{3}$ \\ ${ }^{1}$ Instituto de Física de Cantabria, CSIC-UC, Av. de Los Castros s/n, 39005 Santander, Spain \\ e-mail: manjon@ifca.unican.es \\ 2 Departamento de Física Moderna, Universidad de Cantabria, 39005 Santander, Spain \\ 3 Leiden Observatory, Leiden University, 2300 RA Leiden, The Netherlands
}

Received 14 October 2019 / Accepted 20 May 2020

\begin{abstract}
We performed a free-form strong lensing analysis of the galaxy cluster MACS J1206.2-0847 in order to estimate and constrain its inner dark matter distribution. The free-form method estimates the cluster total mass distribution without using any prior information about the underlying mass. We used 97 multiple lensed images belonging to 27 background sources and derived several models, which are consistent with the data. Among these models, we focus on those that better reproduce the radial images that are closest to the centre of the cluster. These radial images are the best probes of the dark matter distribution in the central region and constrain the mass distribution down to distances $\sim 7 \mathrm{kpc}$ from the centre. We find that the morphology of the innermost radial arcs is due to the elongated morphology of the dark matter halo. We estimate the stellar mass contribution of the brightest cluster galaxy and subtracted it from the total mass in order to quantify the amount of dark matter in the central region. We fitted the derived dark matter density profile with a gNFW, which is characterised by $r_{\mathrm{s}}=167 \mathrm{kpc}, \rho_{\mathrm{s}}=6.7 \times 10^{6} M_{\odot} \mathrm{kpc}^{-3}$, and $\gamma_{\mathrm{gNFW}}=0.70$. These results are consistent with a dynamically relaxed cluster. This inner slope is smaller than the cannonical $\gamma=1$ predicted by standard CDM models. This slope does not favour self-interacting models for which a shallower slope would be expected.
\end{abstract}

Key words. galaxies: clusters: individual: MACS J1206.2-0847 - gravitational lensing: strong - cosmology: observations dark matter - methods: numerical

\section{Introduction}

The measurement of the inner dark matter density profile in galaxy clusters provides a test of dark matter models since these are regions of high density and considerable volume. If dark matter annihilates or interacts with itself, the density in the central region of galaxy clusters is expected to be sensitive to these interactions. The central mass profiles of galaxy clusters can be inferred in several ways, each one probing a different range of radii. These methods include stellar kinematics, strong lensing (SL), weak lensing (WL), Sunyaev-Zeldovich (SZ), and $\mathrm{X}$-ray emission, which cover the distance range from $10 \mathrm{kpc}$ to $1 \mathrm{Mpc}$ scale (Newman et al. 2009; Umetsu et al. 2011; Hogan et al. 2017; Andrade et al. 2019). Strong lensing methods are based on the magnification, shape and, positions of the multiple lensed images of background sources, which are used to set constraints on the mass distribution of the deflector. These images typically appear near the Einstein radius of the lens, or tangential critical curve, which is typically between tens and a few hundred kiloparsecs in galaxy clusters. Arcs can also appear close to the radial critical curve, which can be much closer (a few kiloparsecs) to the centre of the cluster. Constraining the mass profile in the most central region of galaxy clusters is of particular interest since this region contains the largest densities of dark matter. If dark matter has a small cross section for interaction, or if dark matter is ultralight (e.g. in axion-like-particle, or ALP, models), it is at these regions where deviations from pure col- lisionless cold dark matter models are expected (Rocha et al. 2012; Kaplinghat et al. 2013, 2014). In particular, if the cross section for self-interaction is significantly larger than $1 \mathrm{~cm}^{2} \mathrm{~g}^{-1}$, the profile should flatten in the most central region, and offsets between the positions of the brightest cluster galaxy (BCG) and the peak of the dark matter distribution are expected if the cluster is in a merger phase.

The interpretation of strong lensing observations in galaxy clusters can be difficult due to the distortions introduced in the background galaxies and also the lack of information (such as redshifts, Blandford \& Narayan 1992; Schneider et al. 1993; Wambsganss 1998; Narayan \& Bartelmann 1999; Kneib 2002). Fortunately, in recent years, high quality data has enabled us to spectroscopically confirm many families of multiple lensed images, reducing the level of uncertainty and allowing detailed lens reconstructions in many galaxy clusters. Lens reconstruction methods are broadly classified into parametric and free-form methods. Parametric models are the natural choice for modelling strong lensing clusters when the number of lensing constraints is relatively small, such as in the case of QSO lensing, where only three or four multiple images are typically available. These models require that initial assumptions, or priors, about the cluster mass distribution be made. Some of these assumptions are, for instance, that the dark matter haloes follow the luminous matter in the cluster or that galaxy profiles have certain symmetries. However, if the number of lensing constraints is sufficiently high, e.g. a few tens to a hundred, it is possible to accurately 
reconstruct the mass profile of a galaxy cluster, including substructure, in a free-form way. The advantage of a free-form method is that no initial assumptions about the distribution of mass in the galaxy cluster are needed. Lens models derived with free-form methods are useful in these cases where the geometry of the lens is complex, such as in the galaxy cluster MACS0717. In this case, a free-form lens model was able to correctly predict the position of new families of lensed galaxies, which were later confirmed spectroscopically (Diego et al. 2015). Even in cases where the cluster is more regular, free-form methods have proven to be accurate at predicting different observations that were later confirmed. For instance, they predicted the time and position of the reappearance of SN Refsdal correctly (Diego et al. 2016), or the redshift of the family of lensed images in system 7 in the cluster A370 (Diego et al. 2018).

In this work we explored, with strong lensing data alone, the galaxy cluster MACS J1206.2-0847 (hereafter J1206) using the free-form code WSLAP+ (Diego et al. 2005a,b, 2007; Sendra et al. 2014) aiming at estimating its inner total mass distribution (baryonic and dark matter) and constraining the dark matter component. This algorithm does not rely on assumptions on the dark matter distribution and has proven to perform equally well both in morphologically complex clusters (Diego et al. 2015) as well as with more regular clusters (Diego et al. 2016, 2018). This free-form method allows for increased spatial resolution, and flexibility, in certain areas of the lens plane, where more detail is needed. We took advantage of this feature to study the central region of this cluster in greater detail and benefit from the constraints provided by two radial arcs with unusually extended morphology and close to the centre of the cluster. When the performance of WSLAP+ was compared with other codes using simulated observations (Meneghetti et al. 2017), the lens models derived with WSLAP+ reproduced well the lensing potential in those regions where multiple images are present. On the contrary, and not surprisingly, the potential was poorly constrained in those regions without lensing constraints.

The cluster J1206 is at $z=0.439$ and was originally discovered in a short 2 min $R$-band image taken on June 15th 1999 by the University of Hawaii's 2.2-metre telescope (UH2.2m) on Mauna Kea. This observation was triggered by the presence of a X-ray source previously detected by the ROSAT All-Sky Survey (RASS) (RXC J1206.2-0848, Böhringer et al. 2001, 2004). After its discovery, it was included in the MAssive Cluster Survey (MACS), an ongoing project aimed at the compilation of a statistically complete sample of very X-ray luminous (and thus, by inference, massive), distant clusters of galaxies from RASS data (Ebeling et al. 2001, 2007, 2010). The cluster appeared fairly relaxed in its X-ray emission, but had significant amounts of intracluster light which was not centrally concentrated, suggesting that galaxy-scale interactions were still ongoing despite the overall relaxed state (Eichner et al. 2013). The BCG can be easily identified and is located in the centre of the cluster.

Following its initial identification as a potentially massive galaxy cluster, several follow-up observations were performed to confirm its cluster nature and characterise its physical properties. This cluster is actually one of the 25 clusters in the Cluster Lensing and Supernova survey with Hubble (CLASH) programme (Postman et al. 2012), a major project aimed to build a large strong lensing data set on massive relaxed lensing clusters (Zitrin et al. 2012; Umetsu et al. 2012; Biviano et al. 2013). One of the most peculiar lensed images in this cluster is a 15 arcsec long, bright and tangential gravitational arc, extending from a few arcsec to $\approx 20$ arcsec west of the BCG. This arc was spectroscopically observed first by Sand et al. (2004) and later confirmed, with a counter-image, by Ebeling et al. (2009) at $z=1.036$. Ebeling et al. (2009) make an analysis of the total cluster mass distribution as well as of the mass in the cluster core using X-ray, dynamical and strong lensing data. This giant arc and its companion image are treated as a seven-image multiple system in order to perform a strong lensing model. Their strong lensing analysis, based on imaging from the Hubble Space Telescope (HST), yields a mass estimate twice larger than the $\mathrm{X}$-ray mass estimate based on Chandra data. Despite its relaxed appearance at optical and X-ray wavelengths, this discrepancy is believed to be indicative of ongoing merging activity, which is also supported by a small, but significant, offset of the $\mathrm{X}$-ray emission peak from the $\mathrm{BCG}$, and the very high dispersion velocity of the cluster. Zitrin et al. (2012) exploit UV, optical and IR data from the 16 HST bands in the CLASH project along with spectra from VLT/VIMOS in order to spectroscopically identify 47 new multiple lensed images of 12 distant sources. A total of 50 multiple lensed images of 13 sources, spanning redshifts from 1.0 to 5.5 , were known by then. They use 32 secure multiple lensed images belonging to nine sources in a parametric strong lensing reconstruction to derive a detailed mass distribution, and to constrain the inner mass profile of this cluster.

A later comprehensive analysis of this cluster combining weak and strong lensing data from wide-field Subaru imaging and HST observations was performed by Umetsu et al. (2012). They primarly use the parametric strong lens modelling of Zitrin et al. (2012) but also perform some semi-independent free-form strong lensing analyses. They find good agreement in the overlap region between weak and strong lensing constraints. Agreement is found also between the lensing results and X-ray mass measurements, again in the overlap region of the two data sets. Their morphological analysis of both the distribution of galaxies and the reconstructed mass distribution also reveals the presence of an elongated large-scale structure surrounding J1206. This structure runs approximately NW-SE, and it is aligned with the cluster and BCG orientations. The overall shape of J1206 indicates that light follows the mass up to the large scales of the cosmic web probed by observations.

A conclusive assessment on the degree of relaxation of the cluster was finally reached in Biviano et al. (2013) through the analysis of the velocities of several hundreds of cluster members. Building on a data set of $\sim 600$ spectroscopic redshifts of cluster member galaxies out to the cluster outskirts $(\sim 5 \mathrm{Mpc})$, they perform a mass profile reconstruction with galaxy dynamics well beyond the cluster virial radius. The mass profile obtained is found to be in excellent agreement with those derived from all the other independent probes (that is, SL, WL and X-rays) and consistent with a Navarro-Frenk-White (NFW) profile (Navarro et al. 1996, 1997). This agreement lastly indicates that possible systematic biases in dynamical analyses have been properly accounted for, and that the cluster is in fact in a relaxed dynamical state. A pretty good agreement between Chandra $\mathrm{X}$-ray and lensing mass profiles for this cluster is also reported in Donahue et al. (2014). In this case, they use a free-form analysis which combines weak and strong lensing constraints from the HST and from ground-based wide-field data (Merten et al. 2009, 2015).

Finally, the most up-to-date strong lensing analysis of this cluster is performed by Caminha et al. (2017). They were able to identify 23 new spectroscopically confirmed multiple lensed sources, using deep VLT/MUSE (Multi Unit Spectroscopic Explorer) observations in combination with CLASH-VLT imaging (Biviano et al. 2013). Using the positional measurements of 82 spectroscopic multiple images belonging to 27 families and 
a flexible parametric approach, they present an improved measurement of mass distribution in the inner region of J1206. An extension of this strong lensing analysis, including stellar kinematics constraints to model the cluster galaxy population, and using the same catalogue of multiple images and cluster galaxies as in Caminha et al. (2017), is described in Bergamini et al. (2019).

In this paper we present a re-analysis of the latest data on this cluster but with a special emphasis on the central region, that can be well constrained by the two giant radial arcs. Also, the use of a free-form method allows us to explore a wider range of parameters with increased flexibility in the central region through the use of a multiresolution grid (with increased spatial resolution around the BCG). We do this by producing a range of models constrained with the full data set of arc positions, and selecting a posteriori the best models at reproducing the two giant radial arcs.

The structure of this paper is as follows. Section 2 describes the public Hubble imaging and spectroscopic data of both the cluster and the strong lensing images. In Sect. 3 we give a brief description of the lensing reconstruction method used and describe the nine different scenarios assumed to reconstruct the lens and to study the uncertainties and variability in the solutions. In Sect. 4 we expose the results of the lensing analysis, focusing on the reproducibility of two resolved radial arcs near the BCG in order to estimate the amount of dark matter in the very central region. Finally, we conclude in Sect. 5 .

Through the paper, we assume a flat cosmological model with $\Omega_{\mathrm{M}}=0.3, \Lambda=0.7$ and $h=70 \mathrm{~km} \mathrm{~s}^{-1} \mathrm{Mpc}$. For this model, $1 \mathrm{arcsec}=5.68 \mathrm{kpc}$ at the distance of the cluster $(z=0.439)$. The reference point of our system of coordinates is the BCG: $\mathrm{RA}=12: 06: 12.1436$, Dec $=-8: 48: 03.055$ (J2000). In all images (except when noted otherwise), we adopt the standard convention where north is up and east is left.

\section{Data}

We used public imaging data obtained from the ACS and WFC3 Hubble instruments. We retrieved the data from the Mikulski Archive for Space Telescope ${ }^{1}$ (MAST). For the optical data we used filters $F 275 W, F 336 W, F 390 W, F 475 W, F 606 W, F 775 W$, $F 814 W$ and $F 850 L P$ (ID 12069, PI: M. Postman) and for the infrared (IR) data we used filters $F 105 W, F 110 W, F 125 W$, $F 140 W$ and $F 160 W$ (ID 12069, PI: M. Postman). We produced colour images by combining these optical and IR bands.

Regarding the member galaxies in J1206, we restricted ourselves to only those galaxies that have been spectroscopically confirmed. As part of the CLASH-VLT Large Programme, VLT/VIMOS was used to measure redshifts for a sample of 2736 sources over an area of $400 \mathrm{arcmin}^{2}$, spectroscopically confirming 577 galaxy members (Biviano et al. 2013; Rosati et al. $2014)^{2}$. They are defined as the galaxies with a velocity dispersion lower than $\Delta_{v}=2919 \mathrm{~km} \mathrm{~s}^{-1}$ in the cluster rest frame $(z=0.439$, Girardi et al. 2015), which corresponds to a redshift range of $0.425 \leq z \leq 0.453$. Considering among them only those member galaxies that fall within the HST field of view and also are closer to the BCG, we selected the brightest ones and those which are near to strong lensing arcs. The lensing reconstruction method used is only sensitive to galaxies which are really close

\footnotetext{
1 https://archive.stsci.edu/prepds/clash/

2 This spectroscopic catalogue was publicly released in March 2014 and is available at https://archive.stsci.edu/missions/hlsp/ clash/macs1206/catalogs/redshift
}

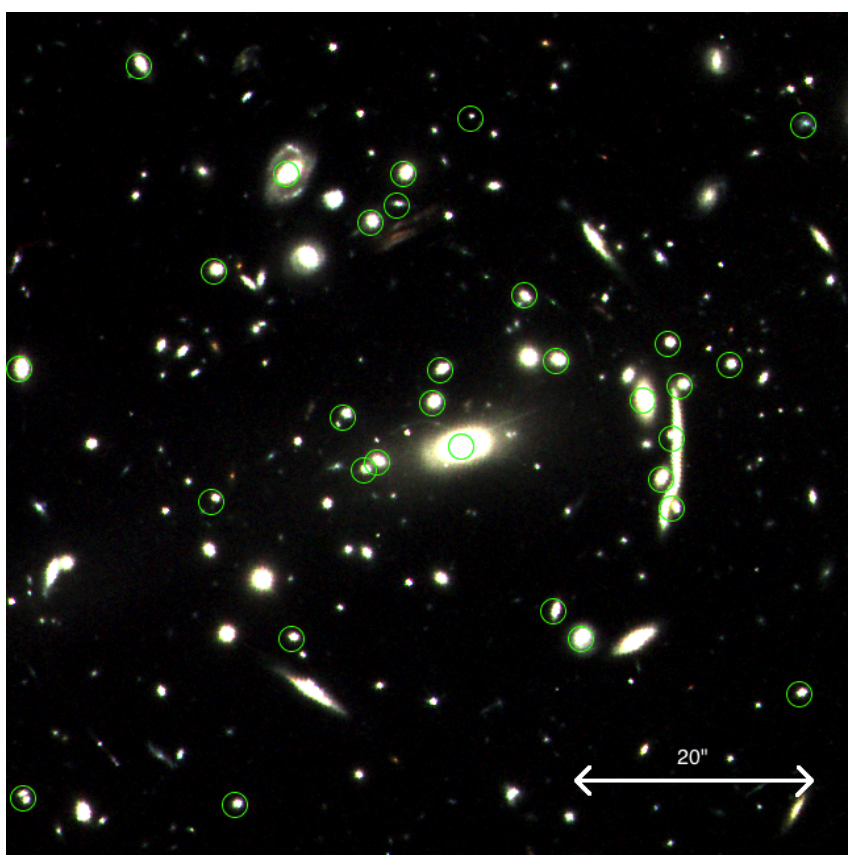

Fig. 1. Image of cluster J1206 showing 31 of the 54 cluster members used in our analysis. The member galaxies that fall within a field of view of 1.18 arcmin centred in the BCG, are marked with green circles.

to an arc (a few arcsec) or that are very massive, and thus its effective radius of influence is larger than a few arcsec. Therefore, only those galaxies which meet these criteria are key for the lensing reconstruction method. The 54 cluster members selected (including the BCG) are listed in Table A.1 and the closest ones to the centre of the cluster are shown in Fig. 1.

For the strong lensing data, we followed the multiple-image system identifications from Caminha et al. (2017), which include 82 spectroscopic multiple images belonging to 27 background sources in the redshift range from 1.01 to 6.06 . They combine spectroscopic measurements from CLASH-VLT (Biviano et al. 2013) and MUSE to find the largest possible number of families of multiple images based on their redshift values. Only multiple images of background galaxies with secure spectroscopic confirmation (identification in the MUSE and HST data) are considered in order to avoid any image misidentification. Multiple images located very close in projection to massive spectroscopically confirmed background galaxies are also discarded because these member galaxies can introduce significant deflections (difficult to model) in addition to those associated with the cluster. From Caminha et al. (2017), we also adopted the numbering scheme (except for some systems we decided to redefine in this work or the systems that include multiple images that were not used for the reasons explained above) as well as the spectroscopic redshifts. In addition to the centroid positions of the multiple lensed systems, we also used the position of individual knots present in well-resolved elongated arcs, that are easily identified thanks to the depth of HST data. The addition of extra knots in well-resolved systems has been proven to greatly improve the accuracy and stability of the derived lensing solutions of the WSLAP+ method (Diego et al. 2016) due to the large extension of the giant arcs. We redefined the 15 arcsec long tangential arc at 20 arcsec west of the BCG $($ ID $=2 b)$ by replacing the original two positions, $2 \mathrm{~b}$ and $2 \mathrm{c}$, (placed in both extremes of this arc) by ten constraints, more or less equally spaced, along the arc. Adding these additional constraints allows 
us to include information on the magnification of giant arcs as the optimisation algorithm tries to focus all knots into a very compact region in the source plane. It is important to emphasise that given the small physical size of the sources, we approximated at first the sources by a point source, so each knot maps to the same location in the source plane. The shape of sources with multiple lensed knots, that are extended in the source plane, is reconstructed through the iterative process in the WSLAP+ method. A first model is run in which the spatial information of sources is not included and fewer iterations are done. Then a second model is run, where the deflection angle map obtained from the first model is used to predict the configuration of the knots in the source plane. The relative position of the knots in the source plane with respect to the centroid of the source is then fixed so only the position of the centroid is a free parameter in the reconstruction. This was done several times increasing the number of iterations at each step until the process ends converging. The shape of extended sources in the source plane was reconstructed by this procedure, avoiding an undesired bias to models which might favour high magnifications due to the initial assumption that the sources are point-like. This technique is used in Diego et al. (2016) for source 1 which have as many as 20 well resolved knots. The configuration of the knots for source 1 is similar to those found by other methods (including parametric ones), suggesting that this iterative process, together with its convergence, is robust against different modelling methodologies. We also redefined the straight arc pointing NW $(\mathrm{ID}=4 \mathrm{~b})$ that arise from the BCG by splitting it into five different knots spread along the arc. On the other hand, counter-images 7c and $7 \mathrm{~d}$, which are 3 arcsec apart, were considered as knots belonging to a unique curved arc leading NE (ID = 7c). Three other knots, adding to a total of five, were included in this curved counterimage. Accordingly, counter-image $7 \mathrm{e}$ was renamed as $7 \mathrm{~d}$. In summary, we use 97 lensing constraints in this work. All these constraints are listed in Table B.1 and the nearest ones to the BCG are shown in Fig. 2.

\section{Lensing reconstruction method}

The lens model for J1206 was obtained using the WSLAP+ code. A very brief description of the method is given in this section. Further details of the method can be found in Diego et al. (2005a, 2007) and Sendra et al. (2014).

WSLAP+ (Weak and Strong Lensing Analysis Package) falls in the category of hybrid methods as it relies on a grid configuration (free-form) plus a compact component that traces the light. The code is able to estimate the cluster mass distribution without using any prior information on the underlying mass provided the number of strong lensing images with known redshifts is sufficiently large. No priors on the shape of the individual haloes are needed. For the usually small compact component, mostly the baryonic contribution, the code assumes that the mass is proportional to the observed flux. All galaxies can be forced to have the same mass-to-light ratio or some galaxies (usually the BCG or other bright galaxies) are allowed to have their own mass-to-light ratio. This method relies on the decomposition of the lens plane into individual cells. We show how the problem in this approximation can be expressed as a system of linear equations, for which a solution can be found.

The fundamental problem in lens modelling is to find out what are the positions of the background sources $\beta$ and the surface mass density $\Sigma(\theta)$ of the lens given a series of lensed images whose positions $\theta$ are known. This is achieved by solving the standard lens equation:

$\beta=\theta-\alpha(\theta, \Sigma(\theta))$

where $\alpha$ is the deflection angle created by the lens. The surface mass density must simultaneously satisfy the lens equation for all images. These lensed images, or strong lensing observables, can be expressed in terms of derivatives of the lensing potential:

$\psi(\theta)=\frac{4 G D_{1} D_{\mathrm{ls}}}{c^{2} D_{\mathrm{s}}} \int \mathrm{d}^{2} \theta^{\prime} \Sigma\left(\theta^{\prime}\right) \ln \left(\left|\theta-\theta^{\prime}\right|\right)$

where $D_{1}, D_{\mathrm{s}}$ and $D_{\mathrm{ls}}$ are the angular diameter distances to the lens, to the source and from the lens to the source, respectively.

The surface mass density can be described by the combination of two components: (i) a soft (or diffuse) component, which we construct as the superposition of $N_{\mathrm{c}}$ Gaussians on a grid of constant width (regular grid) or varying width (adaptive grid), and (ii) a compact component, that accounts for the mass associated with the individual galaxies in the cluster.

Regarding the soft component, other basis functions rather than Gaussians can be chosen (see Diego et al. 2007, for a discussion of alternative basis functions). However, Gaussian functions offer several advantages including a good compromise between the desired compactness and smoothness of the basis function and a fast analytical computation of the integrated mass for a given radius. On the other hand, the compact component was modelled by adopting the light distribution in the filter $F 160 \mathrm{~W}$ and directly assigning to each galaxy a mass proportional to its surface brightness. This mass is later re-adjusted in an optimisation process. The compact component is usually split into independent layers, each one containing one or several cluster members. The separation into layers allows us to constrain differently the mass associated with different kinds of galaxies. This is useful in the case where the light-to-mass ratio may be different, as happens for instance with the BCG.

As in shown in Diego et al. (2005a, 2007), the strong lensing problem can be expressed as a system of linear equations, that can be depicted in the following compact form:

$\Theta=\Gamma X$

where the measured strong lensing observables are contained in the array $\Theta$ of dimension $N_{\theta}=2 N_{\mathrm{SL}}$, the unknown source positions $\beta$ and surface mass density $\Sigma(\theta)$ are contained in the array $X$ of dimension $N_{\mathrm{X}}=N_{\mathrm{c}}+N_{\mathrm{g}}+2 N_{\mathrm{s}}$, and the matrix $\Gamma$, which accounts for all the geometry of the grid, is known (for a given grid configuration and fiducial galaxy deflection field) and has dimension $N_{\Theta} \times N_{\mathrm{X}} . N_{\mathrm{SL}}$ is the number of strong lensing observables (each one contributes with two constraints, $x$, and $y$ ) and $N_{\mathrm{c}}$ is the number of grid points (or cells) that we use to split the field of view in the soft component of the surface mass density. A Gaussian function is placed in each grid point. The width of these Gaussians is chosen in such a way that two nearby grid points with a same amplitude result in a horizontal plateau between the two overlapping Gaussians. In this work, we considered both regular and adaptive (multiresolution) grid configurations. Adaptive grids are useful when there is a clear peak in the mass distribution, for instance here where the cluster has a single well-defined BCG. The regular grid used in this work has $N_{\mathrm{c}}=32 \times 32=1024$ grid points. The multiresolution grid used in this work has $N_{\mathrm{c}}=480$ grid points. These two grids are shown in Fig. 3. $N_{\mathrm{g}}$ is the number of deflection fields (from cluster members) that we consider, that is, the number of mass layers in which we split the compact component. In this 


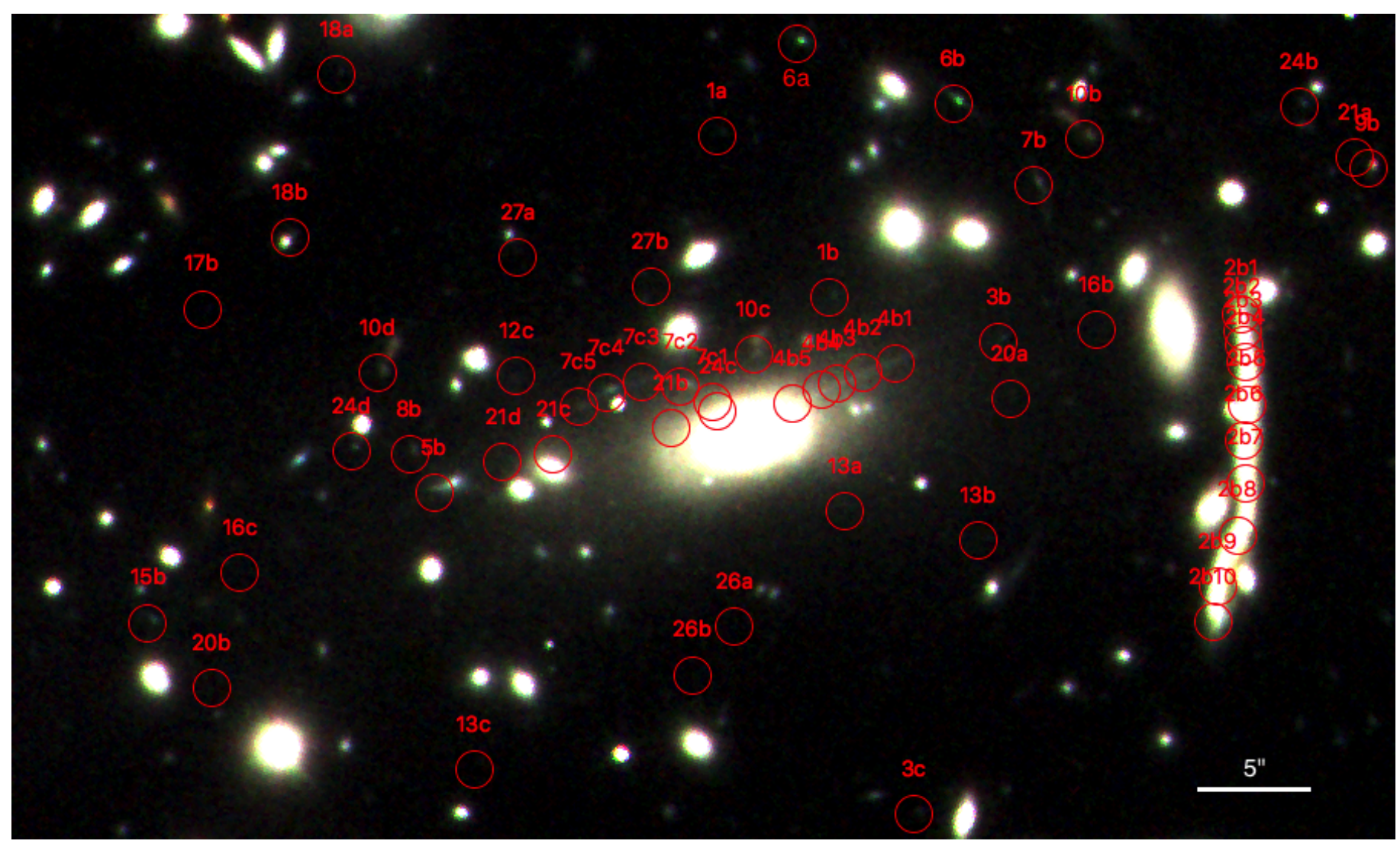

Fig. 2. Image of the cluster J1206, where all the spectroscopically confirmed multiple images used in our strong lensing models, and that fall within a field of view of 58 arcsec, are indicated with red circles.
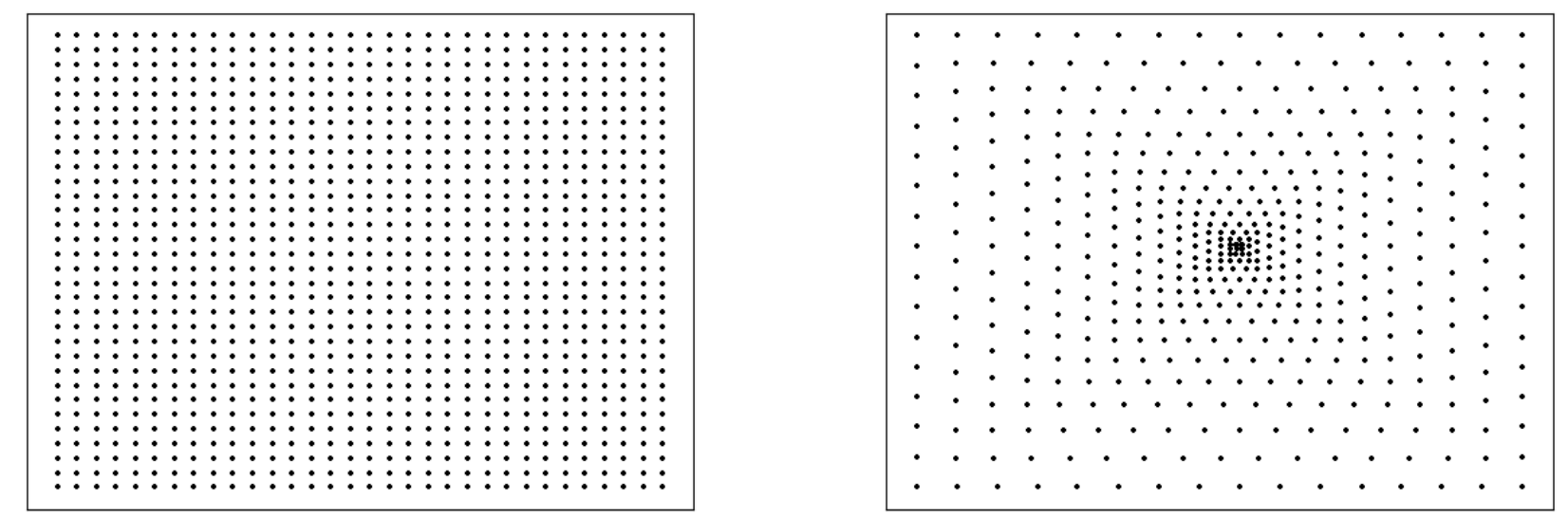

Fig. 3. Depiction of the grids used in this work: a regular grid of 1024 cells $(l e f t)$ and a multiresolution grid of 480 cells (right). The peaks of the individual Gaussians are located at the possitions of the dots.

work, we set $N_{\mathrm{g}}$ equal to 1 or 2 in order to explore different configurations. We contemplated a scenario with $N_{\mathrm{g}}=2$ where all galaxies, except for the BCG, are in the same layer. On the other hand, when $N_{\mathrm{g}}=1$, all the galaxies in the cluster are rescaled by the same parameter because they all are in the same layer. We contemplated four cases with $N_{\mathrm{g}}=1$ : (i) the first one in which all the individual galaxies were assumed to follow the same light-to-mass ratio, (ii) a second case in which the light contribution of the BCG was decreased by a factor of 0.5 , (iii) a third case where the light distribution of the BCG was reduced to one third of its original weight, and (iv) the last case in which the BCG was excluded and only the rest of member galaxies are taken into account. Cases (ii)-(iv) are interesting to explore the possibility that the dark matter content within the BCG is very small and the baryons in the BCG can hide this deficit. We will return to the cases studied at the end of this section. Finally, $N_{\mathrm{s}}$ is the number of background sources (each one contributes with two unknowns, $\beta_{x}$, and $\beta_{y}$ ), which in our case was fixed to 27 families spectroscopically confirmed with CLASH/VLT and MUSE data.

The solution of the system of Eq. (3) is found after minimising a quadratic function of $X$ (as described in Diego et al. 2005a). The minimisation of the quadratic function is done with the constraint that the solution, $X$, must be positive. Since the vector $X$ contains the masses, the renormalisation factors for the galaxy deflection field, and the background source positions, and 
all these quantities are always positive (the reference zero of the source positions is defined in the bottom left corner of the field of view), imposing $X>0$ helps to constrain the space of meaningful solutions and to regularise the solution as it avoids large negative and positive contiguous fluctuations. The quadratic algorithm convergence is fast $(80 \mathrm{~min}$ using the grid with $N_{\mathrm{c}}=1024$ in a $2.7 \mathrm{GHz}$ Intel Core i7 processor) allowing for multiple solutions to be explored in a relatively short time. Different solutions can be obtained after modifying the starting point (or grid configuration) in the optimisation and/or the redshifts of the systems without spectroscopic redshift. A detailed discussion of the quadratic algorithm can be found in Diego et al. (2005a) and a recent discussion about its convergence and performance based on simulated data can be found in Sendra et al. (2014).

In order to account for uncertainties and variability in the lensing models, we explored a range of configurations where we changed the assumptions for the two main components of our method: the light-to-mass ratio of the member galaxies (compact component) and the grid definition (soft component). The number of member galaxies considered was always fixed to 54 . We considered nine types of models, which can be grouped into five categories or cases, according to how the compact component was treated. In case 1 , all galaxies but the BCG were in the same layer $\left(N_{\mathrm{g}}=2\right)$. All galaxies were assumed to follow a fixed light-to-mass ratio while the BCG was allowed to have its own independent light-to-mass ratio. A multiresolution grid with $N_{\mathrm{c}}=480$ cells (1a) and a regular grid of $N_{\mathrm{c}}=1024$ cells (1b) were considered. From case 2 on all the galaxies were in the same layer $\left(N_{\mathrm{g}}=1\right)$. Such as in case 1 , grids with $480(2 \mathrm{a}$, $3 \mathrm{a}, 4 \mathrm{a})$ and 1024 cells $(2 \mathrm{~b}, 3 \mathrm{~b}, 4 \mathrm{~b})$ were again used in cases 2 , 3 , and 4 . In case 2 , galaxies were forced to have the same lightto-mass ratio. In case 3, all galaxies but the BCG had the same light-to-mass ratio equal to one. The BCG was forced to have a mass-to-light ratio of $1 / 2$ times the one for the other member galaxies. Case 4 is similar to case 3 but this time the BCG was forced to have a mass-to-light ratio of $1 / 3$ times the one for the other member galaxies. Finally, in case 5 the BCG was excluded from the compact component and only the grid component contributed with mass in the central region. The other member galaxies had all the same light-to-mass ratio. Only results for the grid with 480 cells (5a) were used in this work ${ }^{3}$.

\section{Results}

We derived a solution for each one of the nine models discussed at the end of Sect. 3 . Solutions typically converge to a stable point after $\approx 10^{5}$ iterations, at which point the minimisation was stopped. We show the critical curves for the two models in case 2, considering a source at redshift $z_{\mathrm{s}}=3$, in Fig. 4. Since most of the differences are due to changes in the grid configuration, the critical curves for the other cases look similar.

To test the quality of the solutions, we compared the observed and predicted counter-images in the image plane. First, we have focused our attention on system 2 since this was the first one spectroscopically observed due to the size and brightness of image $2 \mathrm{~b}$. We used image $2 \mathrm{a}$ to predict the entire system 2 , by delensing $2 \mathrm{a}$ to the source plane and later relensing it back to the image plane. This was done for every lens model considered. The predictions of counter-image $2 b$ for all nine models are shown in Fig. 5. The agreement between the observed and

Regular grid with 1024 cells was also considered but we decided to exclude it due to its clear poor reconstruction of radial arc $7 \mathrm{c}$.

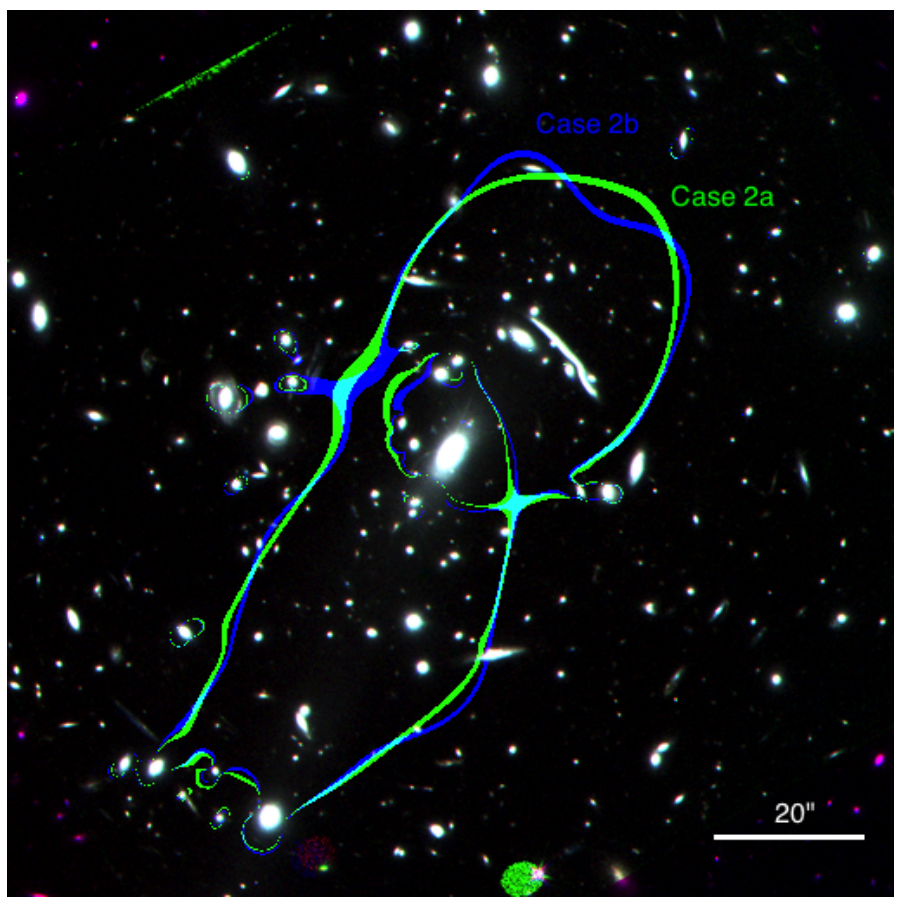

Fig. 4. Critical curves for the two models in case 2 (case 2a in green and case $2 \mathrm{~b}$ in blue), considering a source at $z_{\mathrm{s}}=3$. The field of view is 2 arcmin. The orientation is not north-south, but rotated $44^{\circ}$ counterclockwise.

predicted images is in general good. All predicted images of this arc have the same length as the original one varying a bit in the thickness, straightness and the meandering in the lower region. The fact that all models with different configurations perform well at predicting this counter-image probes the robustness of the models. Cases $3 b$ and $4 b$, and to a lesser degree $1 b$ and $2 b$, are distinctively narrower in the middle section of the arc than the other cases (and the real data), indicating that the predicted radial magnification $\left(\mu_{\mathrm{r}}=(1-\kappa+\gamma)^{-1}\right)$ in this region of the lens plane is smaller for these models. Interestingly, b-type models have a larger contribution from the member galaxies (compact component). The increase in mass in the b-type models is expected since these models correspond to the regular grid, which has a lower resolution around the central region. The impact of a lower resolution grid around the $\mathrm{BCG}$ is evident in case $1 \mathrm{~b}$, in which the mass of the BCG $\left(M_{2}\right.$ in Table 1$)$ is $\approx 3$ times larger than in case 1a. Since b-type models have a larger $\kappa$ around the member galaxies, the value of the radial magnification can be smaller near these galaxies than in a-type models.

Despite the robust prediction of counter-image $2 b$ offered by all models considered, differences arise when looking at their projected density mass profiles. Differences between their profiles are logically greater the closer we approach the centre of the cluster since the very central region is not constrained by any observation. Cases 1 and 2 have density profiles steeper in the inner region as they allow for a greater contribution from the BCG. On the other hand, the density mass profile from case 5, where the BCG was excluded, is shallower. Differences between different grid configurations with the same conditions for the compact component are less significant. We have estimated the values of the logarithmic slope of the projected total mass density profiles for all models within a few kpc from the BCG. The total mass density profiles with the highest (case 1b) and lowest slopes (case 5a) are shown with black dashed-dotted and black 


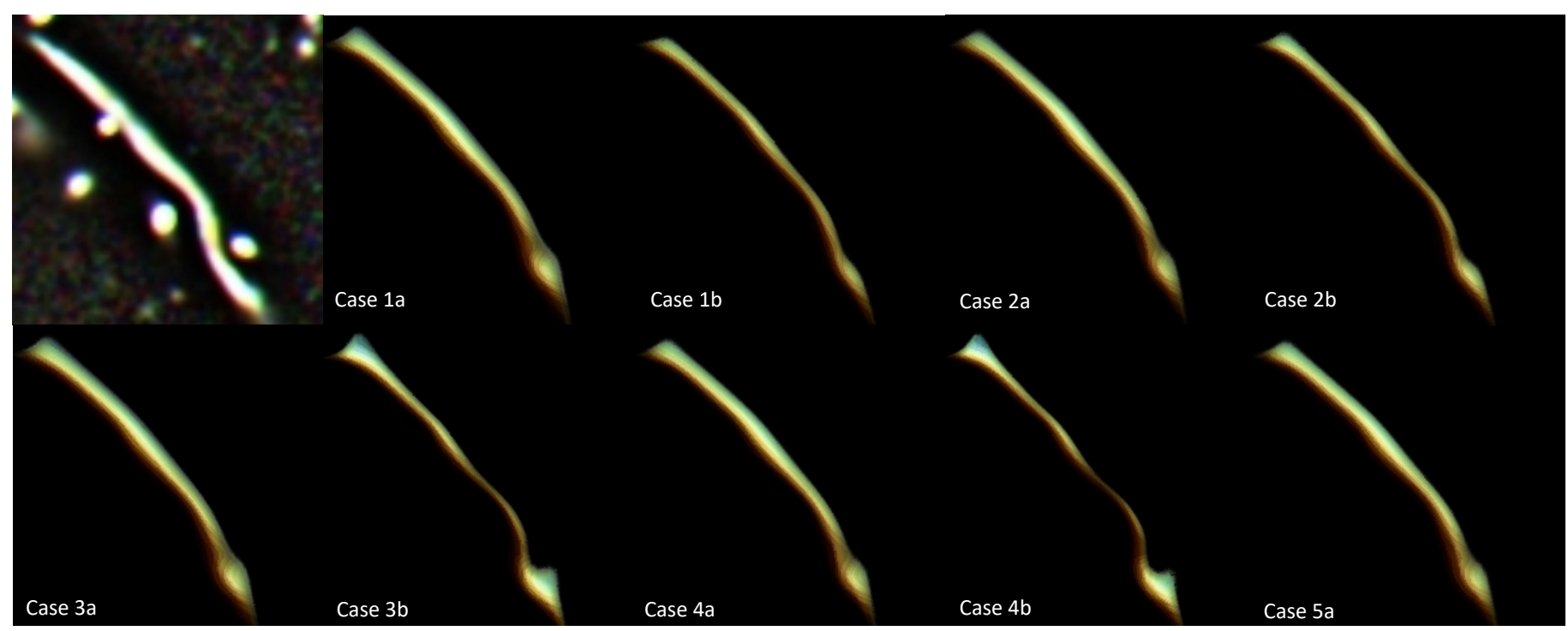

Fig. 5. Original and predicted images of arc $2 b$ using the delensed counter-image $2 a$ as a template of the source for the nine different models discussed at the end of Sect. 3. The field of view and centre of the images are the same in all panels. Image used was filtered to reduce light contribution from member galaxies of the cluster.

Table 1. $\chi^{2}$ values and total mass of the galaxies in the compact component ( $M_{1}$ for layer 1 and $M_{2}$ for layer 2, containing the BCG) for each one of the models considered to the reconstruction of counter-images $4 \mathrm{~b}$ and $7 \mathrm{c}$.

\begin{tabular}{lccccc}
\hline \hline Model & $\chi_{4 \mathrm{~b}}^{2}$ & $\chi_{7 \mathrm{c}}^{2}$ & $\chi_{4 \mathrm{~b}+7 \mathrm{c}}^{2}$ & $M_{1}\left(10^{12} M_{\odot}\right)$ & $M_{2}\left(10^{11} M_{\odot}\right)$ \\
\hline $1 \mathrm{a}$ & 5.6 & 27.2 & 32.8 & 4.31 & 2.54 \\
$1 \mathrm{~b}$ & 12.1 & 21.2 & 33.3 & 3.35 & 8.02 \\
$2 \mathrm{a}$ & 9.4 & 31.3 & 40.7 & 4.54 & - \\
$2 \mathrm{~b}$ & 4.6 & 14.1 & 18.7 & 5.27 & - \\
$3 \mathrm{a}$ & 5.4 & 19.1 & 24.5 & 4.55 & - \\
$3 \mathrm{~b}$ & 1.4 & 24.6 & 26.1 & 7.37 & - \\
$4 \mathrm{a}$ & 5.6 & 22.9 & 28.5 & 4.47 & - \\
$4 \mathrm{~b}$ & 6.2 & 47.1 & 53.3 & 7.57 & - \\
$5 \mathrm{a}$ & 3.3 & 34.4 & 37.7 & 4.32 & - \\
\hline
\end{tabular}

dashed lines, respectively, in Fig. 6. The seven additional models fall in between these two models, and hence for clarity, are not shown in the plot.

The central part of J1206 is the most interesting region we aimed to study. The determination of the total mass distribution in the inner core of J1206 allows us to measure the dark matter density profile and test for possible models of dark matter, such as self-interacting dark matter, that predict shallow or even flat density profiles in the very central region extending up to tens of kpc (and difficult to explain with baryonic feedback). Since the very central region has a large contribution to the mass from the baryonic component itself, one needs to estimate, and later subtract, this baryonic contribution. We used 65 mas CLASH images to measure the photometry of the BCG. The CLASH programme images each cluster with 16 HST filters from UV to near-IR wavelengths. However, due to insufficient overlap between individual exposures, certain filters are prone to cosmic ray contamination. Images from these filters were not included in our analysis. We only measured the photometry from $F 275 W, F 606 W, F 814 W, F 105 W, F 110 W, F 125 W, F 140 W$, and $F 160 W$ images. The photometry of the BCG was measured by running SExtractor in dual-image mode. The $F 160 \mathrm{~W}$ image

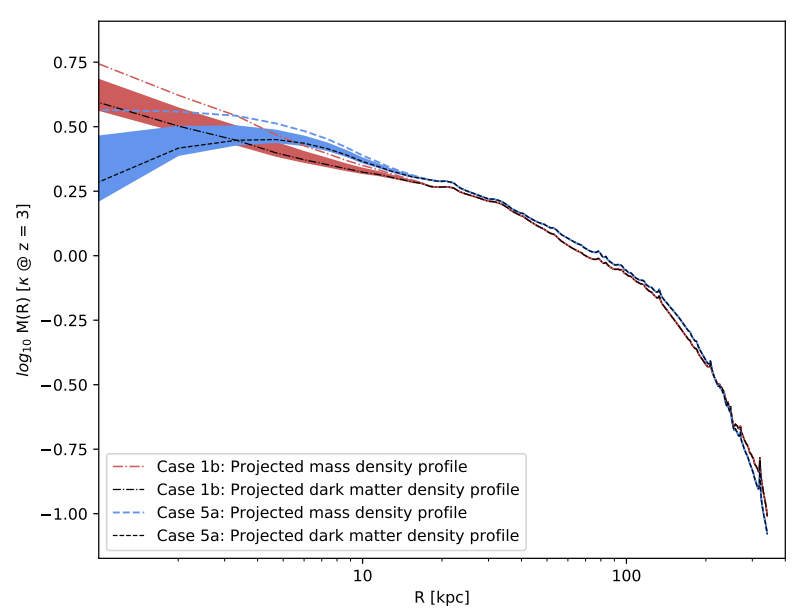

Fig. 6. Mass profile in terms of the critical surface mass density (computed at $z_{\mathrm{s}}=3$ ) for the solutions with the highest (case $1 \mathrm{~b}$ in red) and lowest slopes (case 5a in blue) within a few kpc from the centre of the cluster. The red dash-dotted line and the blue dashed line are the total mass density profiles for each case. The black dash-dotted line surrounded by a red shaded uncertainty region $(3 \sigma)$ and the black dashed line surrounded by a blue shaded uncertainty region $(3 \sigma)$ are the dark matter density profiles in each case. Other models have not been included because their behaviour falls within the borders established by the two models plotted.

was used for source detection, segmentation and the definition of the isophotal apertures. Fluxes and uncertainties in each band were then measured from these isophotal apertures.

We fitted synthetic stellar population spectra to the SED with FAST (Kriek et al. 2009). We made the following assumptions when constructing the synthetic spectra: (i) the Bruzual \& Charlot (2003) stellar population synthesis model, (ii) the Chabrier (2003) initial mass function, (iii) an exponentially declining star formation history, (iv) the Kriek \& Conroy (2013) dust law, (v) a fixed redshift of $z=0.44$, and (vi) a solar metallicity of $Z=0.02$. In addition to these assumptions, the synthetic populations are described by four free parameters, which include stellar mass, star formation timescale, age, and 


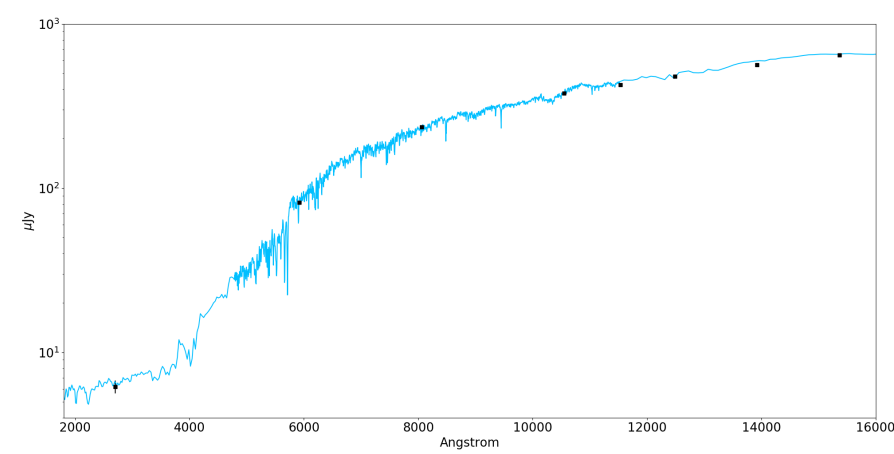

Fig. 7. Best fit of cluster J1206's synthetic stellar population spectrum in logarithmic scale.

extinction. These free parameters are allowed to take on a wide range of physically plausible values. For each combination of their values, a synthetic spectrum was constructed. FAST then calculated the $\chi^{2}$ of each synthetic spectrum to the observed SED, and found the best-fit spectrum, which is shown in Fig. 7. The stellar mass of the best-fit spectrum is $3.61_{-1.98}^{+0.57} \times 10^{11} M_{\odot}$ ( $3 \sigma$ interval). In the end, after removing this stellar mass, we achieved an estimate of the dark matter distribution for each one the models described at the end of Sect. 3. Dark matter density profiles for the models with the highest (case 1b) and lowest slopes (case 5a) are shown with red dashed-dotted and blue dashed lines, respectively, in Fig. 6. It is important to note how case $5 \mathrm{a}$ (which we remind it corresponds to the model where the contribution from the BCG is forced to be zero) results in a declining dark matter density profile at distances below $\approx 3 \mathrm{kpc}$ once the stellar mass is subtracted. This suggests that case $5 \mathrm{a}$ is underestimating the total mass at these radii. However, this is compensated by an excess of mass between $\approx 3 \mathrm{kpc}$ and $\approx 10 \mathrm{kpc}$, keeping a total mass within $10 \mathrm{kpc}$ approximately the same as for the other models. In fact, the integrated total mass within $\leq 10 \mathrm{kpc}$ for case $5 \mathrm{a}\left(6.43 \times 10^{12} M_{\odot}\right)$ is slightly higher than the mass in the same region for the case $1 \mathrm{~b}\left(6.28 \times 10^{12} M_{\odot}\right)$.

\subsection{The central region of $\mathrm{J} 1206$}

As mentioned earlier, we are particularly interested in the central region of J1206. We tested the performance of the nine models by comparing the predicted and observed images of the two resolved radial arcs, $4 \mathrm{~b}$ and $7 \mathrm{c}$, near the BCG. The model that best reproduces these two arcs would be, in principle, the best representation of the dark matter distribution in the centre of J1206. For system 4, we used the counter-image 4c as a template for the source that is delensed and relensed by the nine lens models in order to predict the counter-image $4 \mathrm{~b}$. Counterimage 4a was not used due to its proximity to a cluster member. For system 7 , we considered both counter-image $7 \mathrm{a}$ and $7 \mathrm{~d}$ in the delensing and relensing process for reconstructing arc $7 \mathrm{c}$. Comparing the predictions of arcs $4 \mathrm{~b}$ and $7 \mathrm{c}$ with the data was not trivial in this case since we are making a comparison a posteriori. We did not look for models that minimise the residuals between the observed and predicted images. Instead, we have compared global properties (such as orientation, curvature, and position) of the predicted and observed arcs $4 \mathrm{~b}$ and $7 \mathrm{c}$ after the models were derived using all available information. A classic residual analysis would be, in this case, not optimal since the models were not derived optimising the counter-images $4 \mathrm{~b}$ and $7 \mathrm{c}$ (but optimising the predicted position of all arcs). The metrics used to compare the model with the data are a compromise between the standard

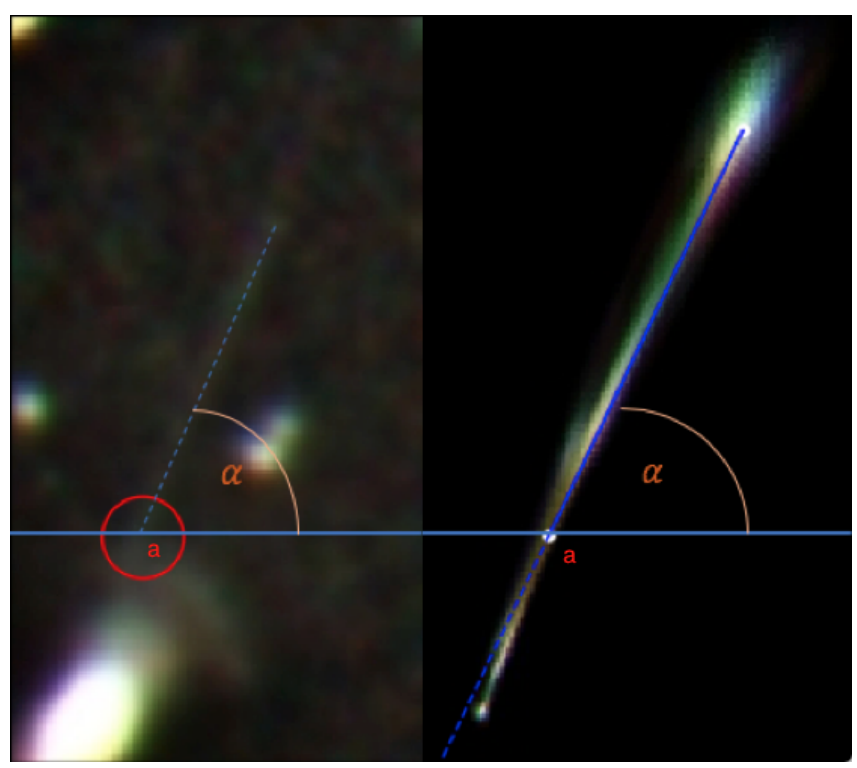

Fig. 8. Left: original counter-image $4 \mathrm{~b}$ where a blue dashed line has been used to approximate its shape, the position where this arc intersects the BCG is surrounded by a red circle (a), and the angle $(\alpha)$ between arc $4 \mathrm{~b}$ and the $x$-axis is shown. Right: counter-image $4 \mathrm{~b}$ predicted with case $2 \mathrm{~b}$ where position (a) is fixed to the same $y$-coordinate selected in the left panel and $x$-coordinate is chosen to correspond with a maximum of the rebuilt image. This counter-image is fitted to a straight line, which has a certain angle $(\alpha)$ with respect to the $x$-axis. This process is repeated for every model considered.

metric, which uses only the position, and more complex figures of merit that could include additional parameters about the morphology of the arcs. We simply added an extra parameter (in addition to the standard position) to account for the main morphological feature in each arc, radius of curvature in one case, and orientation in the other. A more complicated metric could, of course, be chosen but at the expense of introducing unnecessary correlations between the parameters.

First, we focused on the counter-image $4 \mathrm{~b}$ (see Fig. 8), with a shape that can be well approximated by a straight line. From the original counter-image we fitted a straight line defined by the point where the arc intersects the BCG and the angle, $\alpha$, between this line and the $x$-axis (left subfigure). We did the same process for the counter-images predicted using the different models (right subfigure). However, we were not able to select the first point (a) in the same way as we did with the original counterimage because there is no BCG covering the lower part of the predicted image. In order to tackle this we kept the same $y$ coordinate of the first point (a) from the original arc and only looked for the corresponding $x$-coordinate at which the predicted counter-image is maximum. From each one of these fits we computed two values: the $x$-coordinate of the lower point (a) and the angle $(\alpha)$ between the line of fit and the $y$-axis. Explanatory images of the steps followed are shown in Fig. 8. The predictions of counter-image $4 \mathrm{~b}$ for all nine models are shown in Fig. C.1.

For image 7c, we fitted it with a circle. Both from the original counter-image, and from the model-predicted images, we computed the coordinates of the two main knots $\left(7 c_{2}\right.$ and $7 c_{4}$ according to our numbering criterion). We used these two coordinates to fit a circle to the whole arc in the real image and the predicted counter-images and we kept the coordinates of the centre of these circles. From each one of these fits we saved three pairs of values: the coordinates of the knot $7 c_{2}$, the coordinates of the knot $7 c_{4}$ and the coordinates of the centre of the fitted 


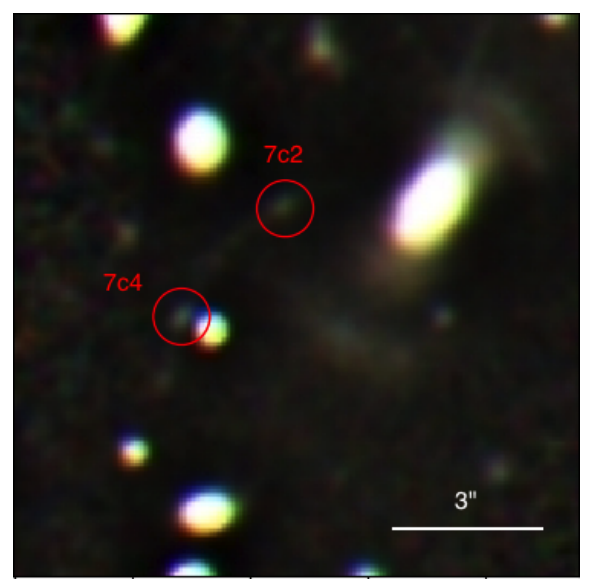

(a)

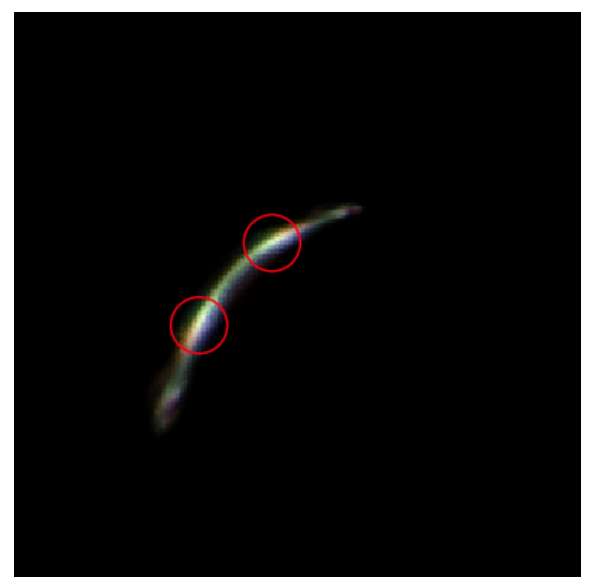

(b)

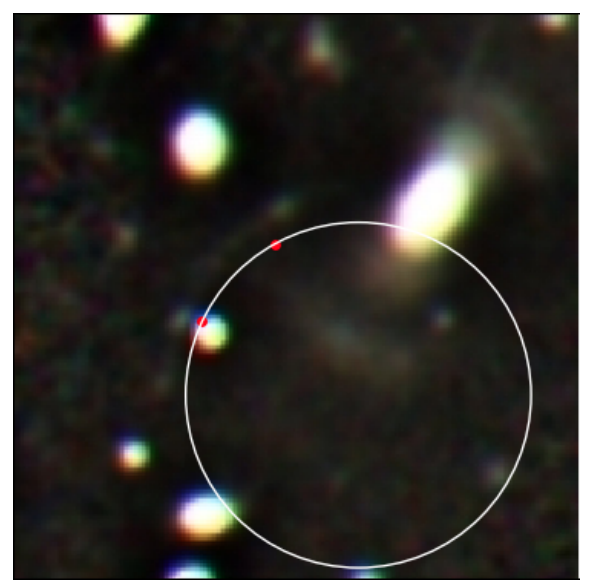

(c)

Fig. 9. Images of the analysis performed with counter-image 7c. (a) Original counter-image $7 \mathrm{c}$ with its two knots, $7 \mathrm{c}_{2}$ and $7 \mathrm{c}_{4}$, indicated with red circles. $(b)$ Counter-image $7 \mathrm{c}$ predicted with case $2 \mathrm{~b}$ with its two knots highlighted. (c) Original counter-image 7c (Fig. 9a) combined with the fit to a circle of the two knots (red dots) from the above prediction (Fig. 9b) so as the shift between both pairs of knots (original and predicted) can be better appreciated.

circle. Explanatory images of the steps followed are shown in Fig. 9. The predictions of counter-image $7 \mathrm{c}$ for all nine models are shown in Fig. C.2.

We finally performed a Pearson's $\chi^{2}$ test statistic in order to compare the performance of the different models in predicting

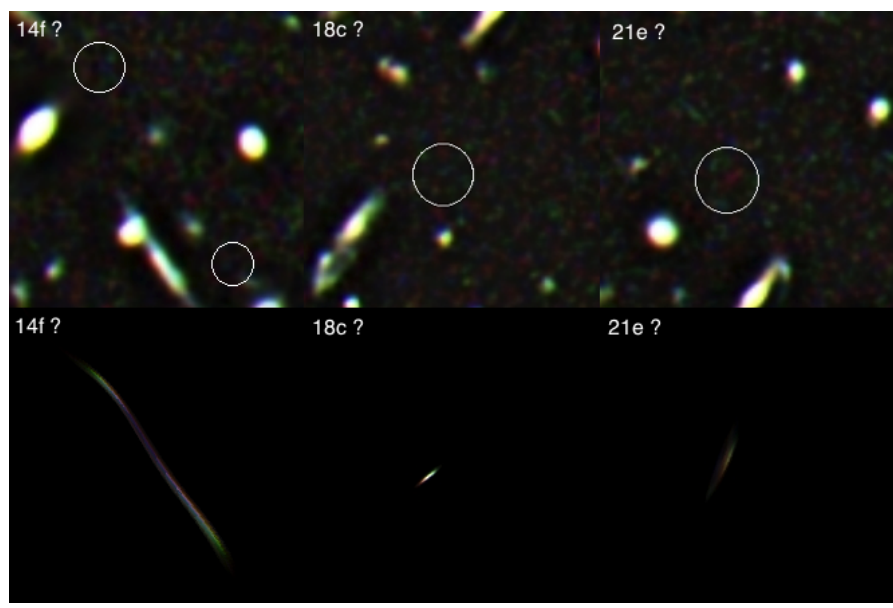

Fig. 10. New counter-images predicted by case $2 b$ for multiple-image systems 14 (left), 18 (middle) and 21 (right). The positions where these counter-images should be seen are indicated with white circles.

counter-images $4 \mathrm{~b}$ and $7 \mathrm{c}$ and to find the models that work better. The values of the five parameters previously saved (two from $4 \mathrm{~b}$ and three from 7c) are then used in the $\chi^{2}$ test. To check for possible systematic effects, the previous process has been repeated several times under different conditions: using different counterimages as templates for the delensing and relensing process, centring the original images in different coordinates or using different filtered images to enhance the signal of the counter-images. Based on $\chi^{2}$ values obtained and shown in Table 1 , case $2 b$ is the model which predicts with more accuracy both radial arcs. If we consider the two radial arcs separately, case $3 \mathrm{~b}$ is the best model describing arc $4 \mathrm{~b}$, while case $2 \mathrm{~b}$ is again the best model at reproducing $7 c$. The worst performers are case $1 b$ for arc $4 b$, case $4 b$ for arc $7 c$, and case $4 \mathrm{~b}$ again for the combination $4 b+7 c$. Interestingly, both the best and worst performers correspond to the category of b-type models, that is models derived with the regular grid. In all cases, the arc $7 \mathrm{c}$ has a $\chi^{2}$ that is significantly worst than arc $4 \mathrm{~b}$, indicating that there is still room for improvement in the model. This can be better appreciated in Fig. 9c, where for the best model, case $2 b$, a small but clear offset between the predicted and observed image can still be seen.

Using case $2 b$, we were able to predict the position of counter-images that were not used in neither our analysis nor in Caminha et al. (2017). Our predictions for these counterimages are consistent with those in Caminha et al. (2017). These counter-images were not taken into account because they might be significantly deflected by massive and nearby early-type galaxies $(22 b, 23 c, 24 \mathrm{e}$ according to the denomination of said paper) or because they are model predicted images in Caminha et al. (2017) but not observed in MUSE nor HST data (4d, 12d, 12e, 13e, 16d, 17c, 17d, 18c, 20c, 20d, $21 \mathrm{e}, 25 \mathrm{c}, 26 \mathrm{c}$ and $27 \mathrm{c}$ according to the denomination of said paper). Case $2 \mathrm{~b}$ also predicts new counter-images for system $14(\mathrm{RA}=12: 06: 15.9459, \mathrm{Dec}=-8: 48: 09.909)$, for system $18(\mathrm{RA}=12: 06: 11.8139, \mathrm{Dec}=-8: 48: 46.595)$, and system $21(\mathrm{RA}=12: 06: 11.2953, \mathrm{Dec}=-8: 48: 40.112)$. These new predicted images are shown in Fig. 10.

\subsection{Mass distribution and mass profile}

The two-dimensional mass distributions of the soft component (grid) for case $2 b$, our best-predict model, and case $3 a$, 
best-predict model using the multirresolution grid, are shown in Fig. 11 and are overlaid with an image of the cluster. The density profile of the galaxy cluster $\mathrm{J} 1206$ from case $2 \mathrm{~b}$, is shown in Fig. 12. Both the projected total mass density profile (blue solid line) and the dark matter density profile (black dotted line) are shown.

The NFW density profile (Navarro et al. 1996, 1997) is the most commonly used profile for modelling the mass distribution of dark matter within large dark matter halos (Bartelmann 1996; Wright \& Brainerd 2000; Golse \& Kneib 2002; Meneghetti et al. 2003). An extension of the NFW density profile, known as the generalised NFW (gNFW) model (see, e.g. Zhao 1996; Jing \& Suto 2000; Wyithe et al. 2001) has been used in this work to fit our estimation of the dark matter density profile within cluster J1206. The density of dark matter as a function of the radius in the gNFW profile is defined as:

$$
\rho_{\mathrm{gNWF}}(r)=\frac{\rho_{\mathrm{s}}}{\left(r / r_{\mathrm{s}}\right)^{\gamma_{\mathrm{gNFW}}}\left(1+r / r_{\mathrm{s}}\right)^{3-\gamma_{\mathrm{gNFW}}}}
$$

where the two parameters, $r_{\mathrm{s}}$ and $\rho_{\mathrm{s}}$, are the scale radius and the characteristic density of the halo, respectively. If $\gamma_{\mathrm{gNFW}}=1$ the original NFW model is recovered, and $\gamma_{\mathrm{gNFW}}<1$ or $\gamma_{\mathrm{gNFW}}>1$ correspond, respectively, to shallower or steeper profiles in the core of J1206.

After assessing the $\chi^{2}$ values of several models to the measured dark matter density profile from case $2 b$, we found a reasonably good fit by using a gNFW density profile with $r_{\mathrm{s}}=167 \mathrm{kpc}$ (virial radius $r_{200}=2 \mathrm{Mpc}$ and concentration parameter $\left.c_{200}=12\right), \rho_{\mathrm{s}}=6.7 \times 10^{6} M_{\odot} \mathrm{kpc}^{-3}$ and $\gamma_{\mathrm{gNFW}}=0.7$. This fit, performed within the range where strong lensing constraints are available, is shown in Fig. 12. These results differ with the ones obtained in previous works with this cluster. The gNFW parameters attained in Caminha et al. (2017) are $r_{\mathrm{s}}=(300 \pm 3) \mathrm{kpc}$, $\rho_{\mathrm{s}}=(1.91 \pm 0.3) \times 10^{6} M_{\odot} \mathrm{kpc}^{-3}$ and $\gamma_{\mathrm{gNFW}}=0.91 \pm 0.04$. The resulting gNFW density profile when using these values is also included in Fig. 12. We note that our profile at radii larger than $200 \mathrm{kpc}$ falls below that of Caminha et al. (2017). Although this may be an artefact in our model due to memory effects of the initial condition (which are more severe in areas where the number of constraints is poor), it may also be a real feature as we are not assuming any specific profile for the halo at large radii. After comparing with the dynamical results from Biviano et al. (2013) shown in Caminha et al. (2017), the dynamical profile falls also faster than the profile in Caminha et al. (2017), and more in line with our profile at large radii. The best-fit NFW model scale radius, virial radius, and concentration parameter obtained in Biviano et al. (2013) by combining the results of two different kinematic methods are $r_{\mathrm{s}}=\left(0.35_{-0.09}^{+0.14}\right) \mathrm{Mpc}, r_{200}=\left(1.96_{-0.09}^{+0.14}\right) \mathrm{Mpc}$, and $c_{200}=(5.6 \pm 1.9)$. This last NFW model is also very close to the best-fit NFW model, with $r_{\mathrm{s}}=(0.34 \pm 0.06) \mathrm{Mpc}$; $r_{200}=(1.96 \pm 0.11) \mathrm{Mpc} ;$ and $c_{200}=(5.8 \pm 1.1)$, obtained by the combined strong and weak lensing analysis published in Umetsu et al. (2012). Our estimate of the scale radius is, therefore, smaller than those of these previous works ${ }^{4}$. Since the number of strong lensing constraints decreases the further we move away from the cluster centre, and there are no constraints beyond $336 \mathrm{kpc}$, our method is not sensitive to the virial radius scale. Accordingly, the boundaries we achieve for the concentration parameter and the scale radius using our data are possibly not

\footnotetext{
4 We also performed a fit to a NFW density profile finding a good fit for $r_{\mathrm{s}}=178$ and $\rho_{\mathrm{s}}=4.56 \times 10^{6} M_{\odot} \mathrm{kpc}^{-3}$. This estimation of the scale radius is smaller than in previous works too.
}

as robust as the ones provided by other data sets, involving for instance weak lensing.

The giant arc $4 \mathrm{~b}$ is particularly interesting since its extreme elongation could in principle be explained by a very shallow profile in the case of a circularly symmetric lens model. This hypothesis is exciting since it could be the result of selfinteractions in the dark matter fluid. Alternatively, the large elongation of arc $4 \mathrm{~b}$ could be explained if the dark matter halo is oriented in the direction of the arc. This would result in a locally shallow potential in that direction, which is the requirement for long elongated radial arcs. When looking at the distribution of dark matter in the central region (see Fig. 11), the dark matter halo has similar morphology, and is oriented in the same direction, as the BCG. That is, the dark matter halo is oriented in the direction of the giant arc $4 \mathrm{~b}$. This fact suggests that one does not need to rely on a cross section for dark matter in order to explain the morphology of arc 4b. When fitting the value of the inner slope assuming a gNFW profile, we obtain a slope of $\gamma=0.7$. This slope is smaller than the canonical $\gamma=1$ predicted by standard CDM models but still relatively far to the expected shallow slope in self-interacting models with cross sections of the order of (or larger than) $1 \mathrm{~cm}^{2} \mathrm{~g}^{-1}$, where one expects $\gamma \approx 0$ (Rocha et al. 2012).

Finally, we found an interesting result in the distribution of sources in the source plane. We estimated the positions of all 27 background galaxies using the lens model $2 \mathrm{~b}$. These galaxies are listed in Table 2, and shown in Fig. 13. Among these, the background galaxies with ID $=4,5,6$, and 7 are all in a narrow redshift interval, $1.424 \leq z \leq 1.426$, and probably forming a group at this redshift since they cluster in the source plane both spatially and in redshift. A reconstructed version of the source plane with these four sources is shown in Fig. 14. We can see how galaxies 4 and 5 are already interacting, and that galaxies 6 and 7 are less than $10 \mathrm{kpc}$ apart, thus in the early phase of an interaction or soon after a first encounter.

\section{Conclusions}

In this work, we presented free-form strong lensing models of the galaxy cluster MACS J1206.2-0847 at $z=0.439$. We used public ACS and WFC3 Hubble data and the code WSLAP+ to derive the lens models. This analysis was built upon spectroscopically confirmed multiple lensed sources identified using public deep MUSE observations in combination with CLASHVLT imaging. Our data set was based on the multiple image identifications from Caminha et al. (2017) but extended with the addition of further knots present in some well-resolved arcs. In total, 97 strong lensing constraints belonging to 27 background sources were used. Several models with different configurations were considered and compared on the basis of how well they were able to reproduce two elongated radial arcs placed close to the BCG. Surprisingly, all models reproduced fairly well these two arcs, independently of the chosen configuration for the grid and of the choice for the mass-to-light ratio of the member galaxies. The geometry of the two giant radial arcs is mostly due to the elongated nature of the dark matter halo, which is reproduced by all models. We estimated, and later subtracted, the stellar baryonic contribution in order to estimate the remaining contribution from the dark matter component in the very central region. After comparing the predicted versus observed central radial images for the range of models, we identified the best model (2b) that reproduces the central potential. This best model is well described by a gNFW profile with $r_{\mathrm{s}}=167 \mathrm{kpc}$ and $\gamma_{\mathrm{gNFW}}=0.7$. Our estimate of the scale radius disagrees with previous results 


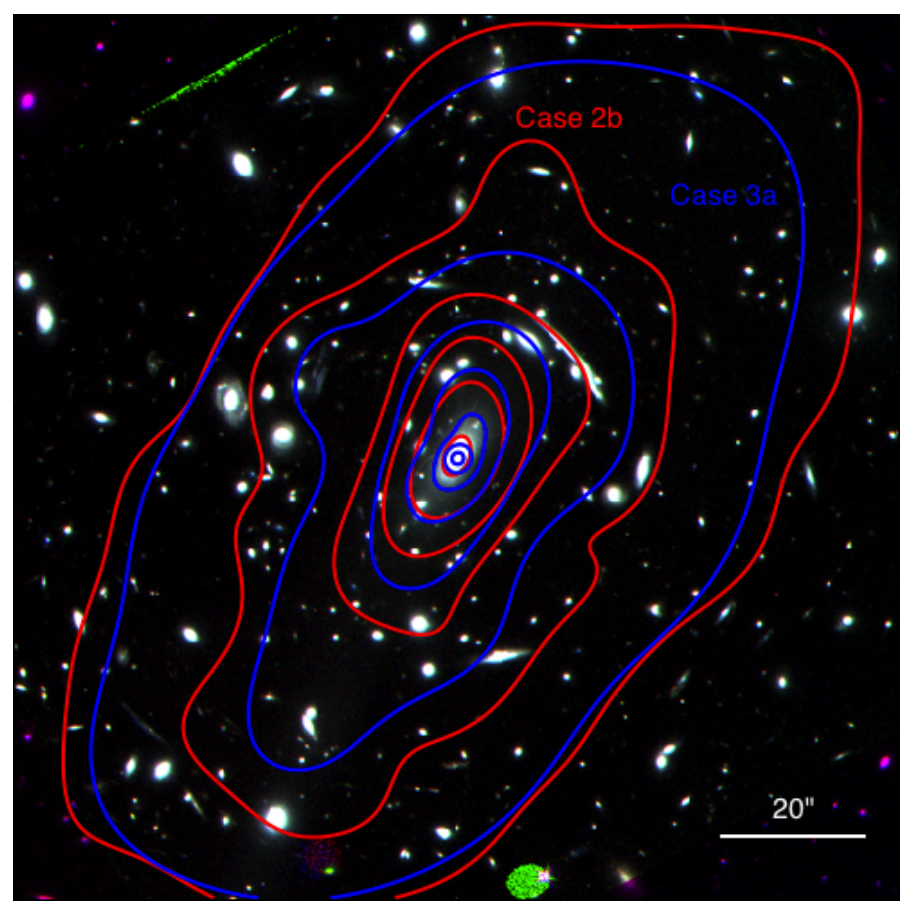

Fig. 11. Contours of the soft component (grid) of the mass distribution for case $2 b$ (in red) and case $3 a$ (in blue) overlapped with an image of the cluster. The orientation of the image is not north-south, but rotated $44^{\circ}$ counterclockwise.

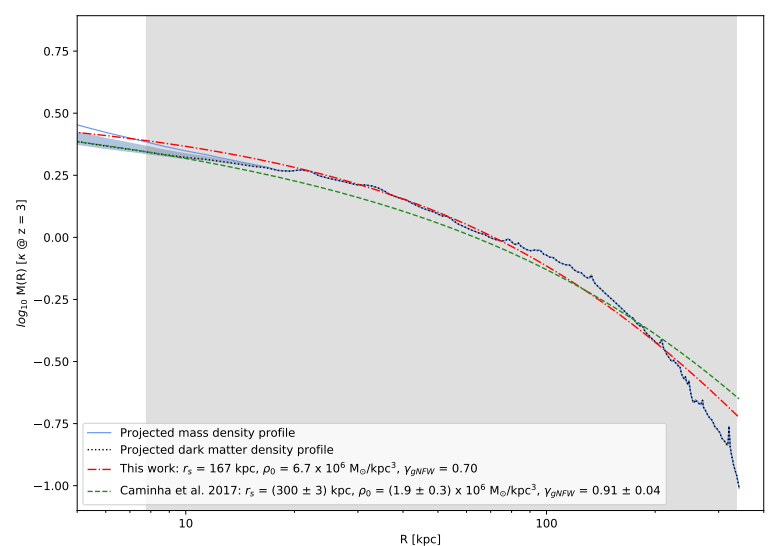

Fig. 12. Mass profile in terms of the critical surface mass density $(\kappa$ computed at $z_{\mathrm{s}}=3$ ) for the model that best reproduces the counterimages $4 b$ and $7 c$ (case $2 b$ ). The blue solid line is the mass density profile calculated with our method while the black dotted line is the dark matter mass density profile estimated after separating the stellar component of the BCG. The blue shaded region represents the $3 \sigma$ uncertainty interval. The red dashed-dotted line is the best fit to a gNFW density profile achieved in this work while the green dashed line corresponds to a gNFW density profile considering the parameters attained in Caminha et al. (2017). The grey shaded area spreading from $7.8 \mathrm{kpc}$ to $335.8 \mathrm{kpc}$ (1.37" to 59.1") covers the region where all the multiple images considered in this work (and listed in Table B.1) are placed.

of the mass distribution within this cluster, although it should be noted that the lack of constraints at large radii impedes a robust estimation of this scale radius. This estimate leads to a concentration parameter $c_{200} \sim 12$, which falls in the range predicted for relaxed clusters. This supports the conclusion from Biviano et al. (2013) that the cluster is in a relaxed dynamical state. The results from our lens modelling are not indicative of a shallow
Table 2. Positions RA and Dec, estimated by case $2 b$, of the 27 background galaxies deflected by the cluster J1206, which have been spectroscopically identified.

\begin{tabular}{lcccc}
\hline \hline $\mathrm{N}^{\circ}$ & RA $(\mathrm{deg})$ & $\sigma_{\mathrm{RA}}(\operatorname{arcsec})$ & Dec $(\mathrm{deg})$ & $\sigma_{\text {Dec }}(\operatorname{arcsec})$ \\
\hline 1 & 181.5502 & 0.1416 & -8.8017 & 0.1105 \\
2 & 181.5488 & 0.0684 & -8.8005 & 0.0540 \\
3 & 181.5501 & 0.0718 & -8.8008 & 0.1029 \\
4 & 181.5508 & 0.0222 & -8.80132 & 0.2144 \\
5 & 181.5509 & 0.0568 & -8.80133 & 0.1017 \\
6 & 181.5499 & 0.1128 & -8.8021 & 0.0911 \\
7 & 181.5500 & 0.2575 & -8.8018 & 0.1000 \\
8 & 181.5509 & 0.0846 & -8.8016 & 0.0275 \\
9 & 181.5488 & 0.0260 & -8.8009 & 0.0426 \\
10 & 181.5503 & 0.0648 & -8.8025 & 0.0978 \\
11 & 181.5553 & 0.0750 & -8.8019 & 0.0728 \\
12 & 181.5496 & 0.0318 & -8.8025 & 0.0105 \\
13 & 181.5515 & 0.1679 & -8.7992 & 0.0839 \\
14 & 181.5572 & 0.0289 & -8.8025 & 0.0695 \\
15 & 181.5518 & 0.0568 & -8.8012 & 0.0392 \\
16 & 181.5512 & 0.0998 & -8.8012 & 0.1452 \\
17 & 181.5511 & 0.0323 & -8.8028 & 0.0076 \\
18 & 181.5506 & 0.0105 & -8.8031 & 0.0396 \\
19 & 181.5548 & 0.0494 & -8.8016 & 0.0166 \\
20 & 181.5517 & 0.0651 & -8.8006 & 0.0427 \\
21 & 181.5496 & 0.0956 & -8.8014 & 0.0651 \\
22 & 181.5477 & 0.0076 & -8.8004 & 0.0033 \\
23 & 181.5552 & 0.0127 & -8.8022 & 0.0868 \\
24 & 181.5499 & 0.0972 & -8.8020 & 0.1815 \\
25 & 181.5527 & 0.0519 & -8.8027 & 0.0407 \\
26 & 181.5511 & 0.0865 & -8.7980 & 0.1390 \\
27 & 181.5493 & 0.0963 & -8.8037 & 0.0730 \\
\hline & & & &
\end{tabular}

Notes. $\sigma_{\mathrm{RA}}$ and $\sigma_{\mathrm{Dec}}$ are the corresponding positional errors for the estimated positions.

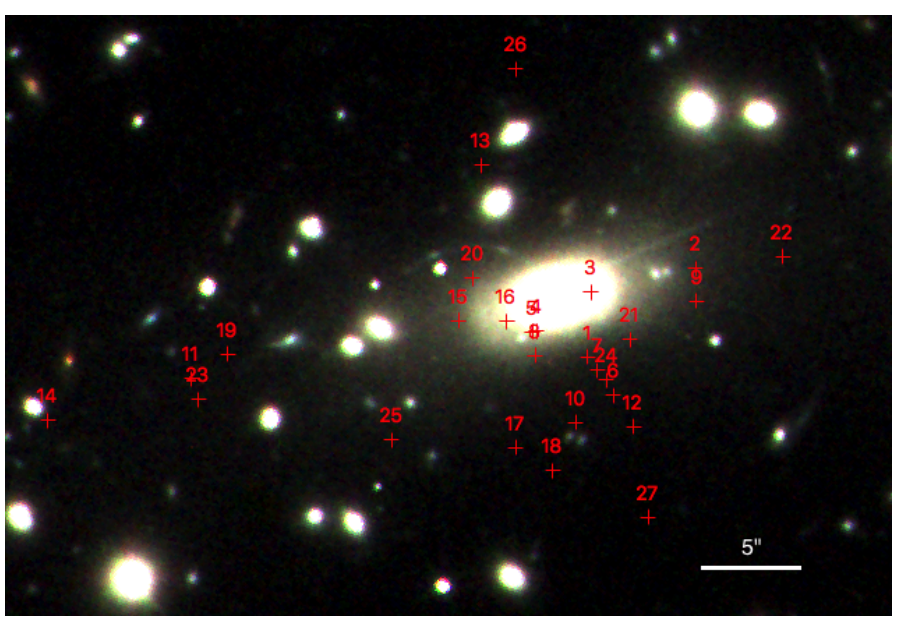

Fig. 13. Image of cluster J1206 showing the positions of all 27 background sources estimated by case $2 \mathrm{~b}$. The field of view has 50 arcsec.

profile in the central region of the cluster, as it would be expected for self-interacting dark matter models. On the contrary, we find a slope close to the expected slope for standard CDM models above $10 \mathrm{kpc}$. This is an important conclusion since our model makes no assumptions about the mass profile in the inner region (that could bias the constraints in the inner slope), other than assuming that the baryonic component traces the luminous mass. 


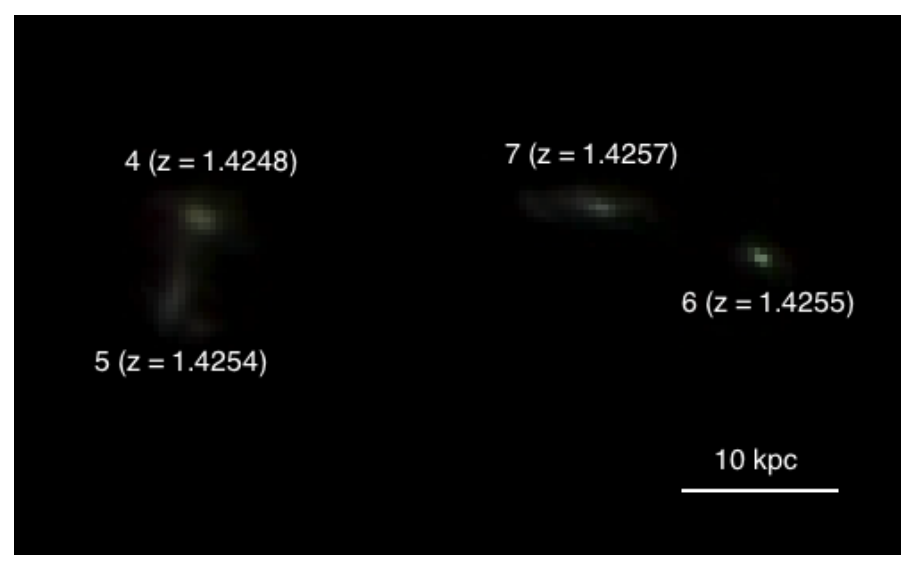

Fig. 14. Reconstructed version of the source plane with background sources 4, 5, 6 and 7, which all have redshifts in the range [1.424-1.426].

Acknowledgements. A.M.G., J.M.D., and D.H. acknowledge the support of project AYA2015-64508-P (MCIU/AEI/MINECO/FEDER, UE) funded by the Ministerio de Economia y Competitividad and project PGC2018-101814-B-100 (MCIU/AEI/MINECO/FEDER, UE) funded by Ministerio de Ciencia, Investigación y Universidades. This research is based on observations made with the NASA/ESA Hubble Space Telescope obtained from the Space Telescope Science Institute, which is operated by the Association of Universities for Research in Astronomy, Inc., under NASA contract NAS 5-26555. We specially thank all people involved in Caminha et al. (2017) for their essential multiple image identification using data taken under the ESO programme IDs 095.A-0181(A), 097.A-0269(A), and 186.A-0798(A); and for making their identifications public. A.M.G. thanks the Department of Physics and Astronomy of the University of Pennsylvania for their hospitality during his stay there, in which he did part of the work shown here. The authors thank the anonymous referee for making valuable suggestions that helped us improve this paper.

\section{References}

Andrade, K. E., Minor, Q., Nierenberg, A., \& Kaplinghat, M. 2019, MNRAS, 487, 1905

Bartelmann, M. 1996, A\&A, 313, 697

Bergamini, P., Rosati, P., Mercurio, A., et al. 2019, A\&A, 631, A16

Biviano, A., Rosati, P., Balestra, I., et al. 2013, A\&A, 558, A1

Blandford, R. D., \& Narayan, R. 1992, ARA\&A, 30, 311

Böhringer, H., Schuecker, P., Guzzo, L., et al. 2001, A\&A, 369, 826

Böhringer, H., Schuecker, P., Guzzo, L., et al. 2004, A\&A, 425, 367

Bruzual, G., \& Charlot, S. 2003, MNRAS, 344, 1000

Caminha, G. B., Grillo, C., Rosati, P., et al. 2017, A\&A, 607, A93

Chabrier, G. 2003, PASP, 115, 763
Diego, J. M., Protopapas, P., Sandvik, H. B., \& Tegmark, M. 2005a, MNRAS, 360, 477

Diego, J. M., Sandvik, H. B., Protopapas, P., et al. 2005b, MNRAS, 362, 1247

Diego, J. M., Tegmark, M., Protopapas, P., \& Sandvik, H. B. 2007, MNRAS 375,958

Diego, J. M., Broadhurst, T., Zitrin, A., et al. 2015, MNRAS, 451, 3920

Diego, J. M., Broadhurst, T., Chen, C., et al. 2016, MNRAS, 456, 356

Diego, J. M., Schmidt, K. B., Broadhurst, T., et al. 2018, MNRAS, 473, 4279

Donahue, M., Mark Voit, G., Mahdavi, A., et al. 2014, ApJ, 74

Ebeling, H., Edge, A. C., \& Henry, J. P. 2001, ApJ, 553, 668

Ebeling, H., Barrett, E., Donovan, D., et al. 2007, ApJ, 661, L33

Ebeling, H., Ma, C., Kneib, J. P., et al. 2009, MNRAS, 395, 1213

Ebeling, H., Edge, A.-C., Mantz, A., et al. 2010, MNRAS, 407, 83

Eichner, T., Setiz, S., Suyu, S. H., et al. 2013, ApJ, 774

Girardi, M., Mercurio, A., Balestra, I., et al. 2015, A\&A, 579, A4

Golse, G., \& Kneib, J.-P. 2002, A\&A, 390, 821

Hogan, M. T., McNamara, B. R., Pulido, F., et al. 2017, ApJ, 837, 51

Jing, Y. P., \& Suto, Y. 2000, ApJ, 529, L69

Kaplinghat, M., Tulin, S., \& Yu, H.-B. 2013, Phys. Rev. Lett., 89, 18

Kaplinghat, M., Keeley, R. E., Linden, T., \& Yu, H.-B. 2014, Phys. Rev. Lett., 113,5

Kneib, J. P. 2002, in The Shapes of Galaxies and Their Dark Halos, ed. P. Natarajan, 50

Kriek, M., \& Conroy, C. 2013, ApJ, 775, L16

Kriek, M., van Dokkum, P. G., Labbé, I., Franx, M., \& Illingworth, G. D. 2009, ApJ, 700, 221

Meneghetti, M., Bartelmann, M., \& Moscardini, L. 2003, MNRAS, 346, 67

Meneghetti, M., Natarajan, P., Coe, D., et al. 2017, MNRAS, 472, 3177

Merten, J., Cacciato, M., Meneghetti, M., Mignone, C., \& Bartelmann, M. 2009, A\&A, 500, 681

Merten, J., Meneghetti, M., Postman, M., et al. 2015, ApJ, 806, A4

Narayan, R., \& Bartelmann, M. 1999, in In Formation of Structure in the Universe. Lectures on Gravitational Lensing, eds. A. Dekel, \& J. P. Ostriker, 360

Navarro, J. F., Frenk, C. S., \& White, S. D. M. 1996, ApJ, 462, 563

Navarro, J. F., Frenk, C. S., \& White, S. D. M. 1997, ApJ, 490, 493

Newman, A. B., Treu, T., Ellis, R. S., et al. 2009, ApJ, 706, 1078

Postman, M., Coe, D., Benítez, N., Bradley, L., \& Broadhurst, T. 2012, ApJS 199, 25

Rocha, M., Peter, A. H. G., Bullock, J. S., et al. 2012, MNRAS, 430, 81

Rosati, P., Balestra, I., Grillo, C., et al. 2014, The Messenger, 158, 48

Sand, D. J., Treu, T., Smith, G. P., \& Ellis, R. 2004, ApJ, 604, 88

Schneider, P., Ehlers, J., \& Falco, E. E. 1993, Gravitational Lenses (SpringerVerlag: New York)

Sendra, I., Diego, J. M., Broadhurst, T., \& Lazkoz, R. 2014, MNRAS, 437, 2642

Umetsu, K., Broadhurst, T., Zitrin, A., Medezinski, E., \& Shu, L.-Y. 2011, ApJ, 729,127

Umetsu, K., Medezinski, E., Nonino, M., et al. 2012, ApJ, 755, 56

Wambsganss, J. 1998, Liv. Rev. Relativ., 1, 12

Wright, C. O., \& Brainerd, T. G. 2000, ApJ, 534, 54

Wyithe, J. S. B., Turner, E. L., \& Spergel, D. N. 2001, ApJ, 555, 504

Zhao, H. 1996, MNRAS, 278, 488

Zitrin, A., Rosati, P., Nonino, M., et al. 2012, ApJ, 749, 97 


\section{Appendix A: Member galaxies of the cluster}

Table A.1. Cluster member galaxies in J1206 taken into account in our strong lensing analysis.

\begin{tabular}{|c|c|c|}
\hline $\mathrm{N}^{\circ}$ & RA & Dec \\
\hline 1 & 12:06:08.0928 & $-8: 48: 23.821$ \\
\hline 2 & 12:06:08.5999 & $-8: 48: 12.416$ \\
\hline 3 & 12:06:08.6263 & $-8: 47: 54.737$ \\
\hline 4 & 12:06:08.6426 & $-8: 48: 26.946$ \\
\hline 5 & 12:06:08.7348 & $-8: 47: 33.508$ \\
\hline 6 & 12:06:09.1373 & $-8: 47: 23.132$ \\
\hline 7 & $12: 06: 09.3401$ & $-8: 47: 21.786$ \\
\hline 8 & 12:06:09.8885 & $-8: 47: 32.024$ \\
\hline 9 & 12:06:09.9206 & $-8: 48: 27.414$ \\
\hline 10 & 12:06:10.3814 & $-8: 47: 55.388$ \\
\hline 11 & 12:06:10.6385 & $-8: 48: 56.372$ \\
\hline 12 & 12:06:10.7038 & $-8: 47: 57.444$ \\
\hline 13 & 12:06:10.7573 & $-8: 48: 09.302$ \\
\hline 14 & $12: 06: 10.7623$ & $-8: 48: 02.588$ \\
\hline 15 & 12:06:10.7856 & $-8: 47: 53.322$ \\
\hline 16 & 12:06:10.8290 & $-8: 48: 06.570$ \\
\hline 17 & 12:06:10.9505 & $-8: 47: 58.855$ \\
\hline 18 & 12:06:10.9543 & $-8: 47: 12.203$ \\
\hline 19 & 12:06:11.3618 & $-8: 48: 22.100$ \\
\hline 20 & 12:06:11.4444 & $-8: 48: 56.200$ \\
\hline 21 & $12: 06: 11.5236$ & $-8: 47: 54.982$ \\
\hline 22 & $12: 06: 11.5370$ & $-8: 48: 19.361$ \\
\hline 23 & $12: 06: 11.7094$ & $-8: 47: 14.748$ \\
\hline 24 & $12: 06: 11.7270$ & $-8: 47: 48.536$ \\
\hline 25 & 12:06:12.0842 & $-8: 47: 31.330$ \\
\hline 26 & 12:06:12.1459 & $-8: 48: 03.316$ \\
\hline 27 & $12: 06: 12.2818$ & $-8: 47: 55.864$ \\
\hline 28 & $12: 06: 12.3353$ & $-8: 47: 59.064$ \\
\hline 29 & 12:06:12.5191 & $-8: 47: 36.737$ \\
\hline 30 & $12: 06: 12.5676$ & $-8: 47: 39.793$ \\
\hline 31 & $12: 06: 12.6288$ & $-8: 48: 52.153$ \\
\hline 32 & $12: 06: 12.7015$ & $-8: 48: 04.831$ \\
\hline 33 & $12: 06: 12.7282$ & $-8: 46: 48.205$ \\
\hline 34 & $12: 06: 12.7418$ & $-8: 47: 41.503$ \\
\hline 35 & 12:06:12.7493 & $-8: 48: 52.625$ \\
\hline 36 & $12: 06: 12.7822$ & $-8: 48: 05.627$ \\
\hline 37 & 12:06:12.9187 & $-8: 48: 00.443$ \\
\hline 38 & 12:06:13.2612 & $-8: 48: 21.964$ \\
\hline 39 & 12:06:13.2898 & $-8: 47: 36.773$ \\
\hline 40 & $12: 06: 13.6320$ & $-8: 48: 38.160$ \\
\hline 41 & $12: 06: 13.7657$ & $-8: 47: 46.172$ \\
\hline 42 & 12:06:13.7846 & $-8: 48: 08.730$ \\
\hline 43 & $12: 06: 14.2651$ & $-8: 47: 26.182$ \\
\hline 44 & 12:06:14.5106 & $-8: 48: 46.404$ \\
\hline 45 & $12: 06: 15.0310$ & $-8: 48: 37.505$ \\
\hline 46 & $12: 06: 15.0482$ & $-8: 47: 55.752$ \\
\hline 47 & 12:06:15.3494 & $-8: 47: 43.267$ \\
\hline 48 & $12: 06: 15.3562$ & $-8: 47: 43.440$ \\
\hline 49 & 12:06:15.6612 & $-8: 48: 21.823$ \\
\hline 50 & $12: 06: 15.7313$ & $-8: 48: 12.200$ \\
\hline 51 & $12: 06: 15.9929$ & $-8: 48: 17.233$ \\
\hline 52 & $12: 06: 16.0973$ & $-8: 48: 06.260$ \\
\hline 53 & 12:06:16.0990 & $-8: 48: 11.948$ \\
\hline 54 & 12:06:16.2806 & $-8: 48: 02.956$ \\
\hline
\end{tabular}




\section{Appendix B: Multiple images}

Table B.1. Spectroscopically identified multiple images in J1206 taken into account in our strong lensing analysis.

\begin{tabular}{|c|c|c|c|}
\hline ID & RA & Dec & $z_{\mathrm{s}}$ \\
\hline $1 \mathrm{a}$ & $12: 06: 1$ & $-8: 47: 50.7192$ & 1.0121 \\
\hline $1 b$ & 12:06:11.9050 & $-8: 47: 57.4584$ & 1.0121 \\
\hline $1 \mathrm{c}$ & $12: 06: 11.7288$ & $-8: 48: 23.9580$ & 1.0121 \\
\hline $2 \mathrm{a}$ & $12: 06: 11.2296$ & $-8: 47: 44.4480$ & 1.0369 \\
\hline $2 b_{1}$ & $12: 06: 10.7420$ & $-8: 47: 58.1560$ & 1.0369 \\
\hline $2 b_{2}$ & 12:06:10.7396 & $-8: 47: 58.9030$ & 1.0369 \\
\hline $2 b_{3}$ & 12:06:10.7358 & $-8: 47: 59.4920$ & 1.0369 \\
\hline $2 b_{4}$ & 12:06:10.7304 & $-8: 48: 00.1940$ & 1.0369 \\
\hline $2 b_{5}$ & 12:06:10.7268 & $-8: 48: 01.9390$ & 1.0369 \\
\hline $2 b_{6}$ & $12: 06: 10.7358$ & $-8: 48: 03.4150$ & 1.0369 \\
\hline $2 b_{7}$ & $12: 06: 10.7322$ & $-8: 48: 05.2050$ & 1.0369 \\
\hline $2 b_{8}$ & 12:06:10.7541 & $-8: 48: 07.3880$ & 1.0369 \\
\hline $2 b_{9}$ & 12:06:10.8112 & $-8: 48: 09.5570$ & 1.0369 \\
\hline $2 b_{10}$ & $12: 06: 10.8245$ & $-8: 48: 10.9872$ & 1.0369 \\
\hline $3 a$ & $12: 06: 12.1368$ & $-8: 47: 44.0448$ & 1.0433 \\
\hline $3 b$ & $12: 06: 11.4266$ & $-8: 47: 59.3196$ & 1.0433 \\
\hline $3 \mathrm{c}$ & $12: 06: 11.6657$ & $-8: 48: 19.0116$ & 1.0433 \\
\hline $4 a$ & $12: 06: 12$ & $-8: 47: 4$ & 1.4248 \\
\hline $4 b_{1}$ & $12: 06: 1$ & $-8: 48: 00.2052$ & 1.4248 \\
\hline $4 b_{2}$ & 12:06:11.8088 & $-8: 48: 00.6250$ & 1.4248 \\
\hline $4 b_{3}$ & $12: 06: 11.8837$ & $-8: 48: 01.0500$ & 1.4248 \\
\hline $4 b_{4}$ & $12: 06: 11.9246$ & $-8: 48: 01.2810$ & 1.4248 \\
\hline $4 b_{5}$ & $12: 06: 12.0083$ & $-8: 48: 01.9110$ & 1.4248 \\
\hline $4 c$ & 12:06:11.9405 & $-8: 48: 28.6740$ & 1.4248 \\
\hline $5 a$ & $12: 06: 12.8537$ & $-8: 47: 42.6804$ & 1.4254 \\
\hline $5 b$ & 12:06:13.0169 & $-8: 48: 05.5872$ & 1.4254 \\
\hline $5 c$ & $12: 06: 12.0012$ & $-8: 48: 29.1528$ & 1.4254 \\
\hline $6 a$ & $12: 06: 1$ & $-8: 47: 46.9032$ & 1.4255 \\
\hline $6 b$ & 12:06:11.5534 & $-8: 47: 49.4088$ & 1.4255 \\
\hline $6 c$ & $12: 06: 11.5320$ & $-8: 48: 33.4188$ & 1.4255 \\
\hline $7 a$ & $12: 06: 12.1351$ & $-8: 47: 44.5344$ & 1.4257 \\
\hline $7 b$ & $12: 06: 11.3263$ & $-8: 47: 52.7928$ & 1.4257 \\
\hline $7 c_{1}$ & $12: 06: 12.2328$ & $-8: 48: 01.8410$ & 1.4257 \\
\hline $7 c_{2}$ & $12: 06: 12.3214$ & $-8: 48: 01.1808$ & 1.4257 \\
\hline $7 c_{3}$ & $12: 06: 12.4327$ & $-8: 48: 00.9640$ & 1.4257 \\
\hline $7 c_{4}$ & $12: 06: 12.5311$ & $-8: 48: 01.4328$ & 1.4257 \\
\hline $7 c_{5}$ & $12: 06: 12.6092$ & $-8: 48: 02.0260$ & 1.4257 \\
\hline $7 d$ & 12:06:11.5937 & $-8: 48: 31.8816$ & 1.4257 \\
\hline $8 \mathrm{a}$ & $12: 06: 12.8777$ & $-8: 47: 44.7216$ & 1.4864 \\
\hline $8 b$ & $12: 06: 13.0858$ & $-8: 48: 03.9744$ & 1.4864 \\
\hline $8 c$ & 12:06:11.9897 & $-8: 48: 31.9932$ & 1.4864 \\
\hline $9 a$ & $12: 06: 11.2178$ & $-8: 47: 35.3184$ & 1.9600 \\
\hline $9 b$ & 12:06:10.3855 & $-8: 47: 52.1232$ & 1.9600 \\
\hline $9 \mathrm{c}$ & 12:06:10.6507 & $-8: 48: 26.9496$ & 1.9600 \\
\hline $10 \mathrm{a}$ & $12: 06: 12.5880$ & $-8: 47: 42.0036$ & 2.5393 \\
\hline $10 \mathrm{~b}$ & $12: 06: 11.1850$ & $-8: 47: 50.8740$ & 2.5393 \\
\hline $10 \mathrm{c}$ & $12: 06: 12.1169$ & $-8: 47: 59.8452$ & - \\
\hline
\end{tabular}

Table B.1. continued.

\begin{tabular}{|c|c|c|c|}
\hline ID & RA & Dec & $z_{\mathrm{s}}$ \\
\hline $10 \mathrm{~d}$ & 12:06:13.1746 & $-8: 48: 00.5760$ & - \\
\hline $10 \mathrm{e}$ & 12:06:11.7185 & $-8: 48: 42.5268$ & - \\
\hline $11 \mathrm{a}$ & $12: 06: 15.0370$ & $-8: 47: 48.0192$ & 3.0358 \\
\hline $11 \mathrm{~b}$ & $12: 06: 14.9988$ & $-8: 48: 17.6796$ & 3.0358 \\
\hline $11 \mathrm{c}$ & 12:06:14.5375 & $-8: 48: 32.3568$ & 3.0358 \\
\hline $12 \mathrm{a}$ & $12: 06: 11.6717$ & $-8: 47: 37.3812$ & 3.3890 \\
\hline $12 b$ & 12:06:11.0690 & $-8: 47: 43.3932$ & 3.3890 \\
\hline $12 c$ & $12: 06: 12.7843$ & $-8: 48: 00.7092$ & 3.3890 \\
\hline $13 \mathrm{a}$ & 12:06:11.8598 & $-8: 48: 06.3648$ & 3.3961 \\
\hline $13 b$ & 12:06:11.4862 & $-8: 48: 07.6140$ & 3.3961 \\
\hline $13 c$ & 12:06:12.9048 & $-8: 48: 17.1324$ & 3.3961 \\
\hline $13 \mathrm{~d}$ & $12: 06: 12.3334$ & $-8: 48: 24.7104$ & 3.3961 \\
\hline $14 \mathrm{a}$ & $12: 06: 15.9739$ & $-8: 48: 16.1280$ & 3.7531 \\
\hline $14 b$ & $12: 06: 15.9540$ & $-8: 48: 17.0388$ & 3.7531 \\
\hline $14 \mathrm{c}$ & $12: 06: 15.9540$ & $-8: 48: 18.5292$ & 3.7531 \\
\hline $14 d$ & 12:06:15.9060 & $-8: 48: 22.7808$ & 3.7531 \\
\hline $14 \mathrm{e}$ & 12:06:15.7418 & $-8: 48: 27.6840$ & 3.7531 \\
\hline $15 \mathrm{a}$ & $12: 06: 13.4309$ & $-8: 47: 29.8860$ & 3.7611 \\
\hline $15 b$ & $12: 06: 13.8240$ & $-8: 48: 11.0016$ & 3.7611 \\
\hline $15 \mathrm{c}$ & $12: 06: 12.4195$ & $-8: 48: 39.4704$ & - \\
\hline $16 a$ & 12:06:13.1002 & $-8: 47: 28.3272$ & 3.7617 \\
\hline $16 b$ & $12: 06: 11.1516$ & $-8: 47: 58.8156$ & 3.7617 \\
\hline $16 c$ & 12:06:13.5648 & $-8: 48: 08.8956$ & 3.7617 \\
\hline $17 \mathrm{a}$ & $12: 06: 13.4726$ & $-8: 47: 44.2320$ & 3.8224 \\
\hline $17 b$ & 12:06:13.6699 & $-8: 47: 57.9192$ & 3.8224 \\
\hline $18 \mathrm{a}$ & 12:06:13.2902 & $-8: 47: 48.1704$ & 4.0400 \\
\hline $18 \mathrm{~b}$ & $12: 06: 13.4225$ & $-8: 47: 54.9420$ & 4.0400 \\
\hline $19 a$ & 12:06:14.9002 & $-8: 47: 41.5500$ & 4.0520 \\
\hline $19 b$ & 12:06:14.8495 & $-8: 48: 18.8604$ & 4.0520 \\
\hline $19 \mathrm{c}$ & 12:06:14.3491 & $-8: 48: 34.0668$ & 4.0520 \\
\hline $20 \mathrm{a}$ & $12: 06: 11.3933$ & $-8: 48: 01.7136$ & 4.0553 \\
\hline $20 \mathrm{~b}$ & 12:06:13.6414 & $-8: 48: 13.7268$ & 4.0553 \\
\hline $21 \mathrm{a}$ & $12: 06: 10.4234$ & $-8: 47: 51.6264$ & 4.0718 \\
\hline $21 b$ & 12:06:12.3509 & $-8: 48: 02.9304$ & 4.0718 \\
\hline $21 c$ & $12: 06: 12.6840$ & $-8: 48: 04.0140$ & 4.0718 \\
\hline $21 d$ & $12: 06: 12.8232$ & $-8: 48: 04.3344$ & 4.0718 \\
\hline $22 \mathrm{a}$ & 12:06:10.6387 & $-8: 47: 29.1048$ & 4.2913 \\
\hline $22 b$ & 12:06:9.8122 & $-8: 48: 21.9384$ & 4.2913 \\
\hline $23 \mathrm{a}$ & 12:06:15.1805 & $-8: 47: 48.8148$ & 4.7293 \\
\hline $23 b$ & $12: 06: 15.2489$ & $-8: 48: 13.2120$ & 4.7293 \\
\hline $24 \mathrm{a}$ & $12: 06: 12.3307$ & $-8: 47: 28.6800$ & 5.6984 \\
\hline $24 b$ & 12:06:10.5804 & $-8: 47: 49.5384$ & 5.6984 \\
\hline $24 \mathrm{c}$ & 12:06:12.2186 & $-8: 48: 02.1852$ & 5.6984 \\
\hline $24 d$ & $12: 06: 13.2504$ & $-8: 48: 03.8376$ & 5.6984 \\
\hline $25 a$ & $12: 06: 14.3314$ & $-8: 47: 47.6232$ & 5.7927 \\
\hline $25 b$ & $12: 06: 14.4245$ & $-8: 48: 00.6372$ & 5.7927 \\
\hline $26 a$ & 12:06:12.1706 & $-8: 48: 11.2032$ & 6.0106 \\
\hline $26 b$ & $12: 06: 12.2906$ & $-8: 48: 13.2048$ & 6.0106 \\
\hline $27 \mathrm{a}$ & $12: 06: 12.7802$ & $-8: 47: 55.7700$ & 6.0601 \\
\hline $27 \mathrm{~b}$ & 12:06:12.4058 & $-8: 47: 57.0012$ & 6.0601 \\
\hline
\end{tabular}




\section{Appendix C: Model-predicted counter-images $4 \mathrm{~b}$ and $7 \mathrm{c}$}

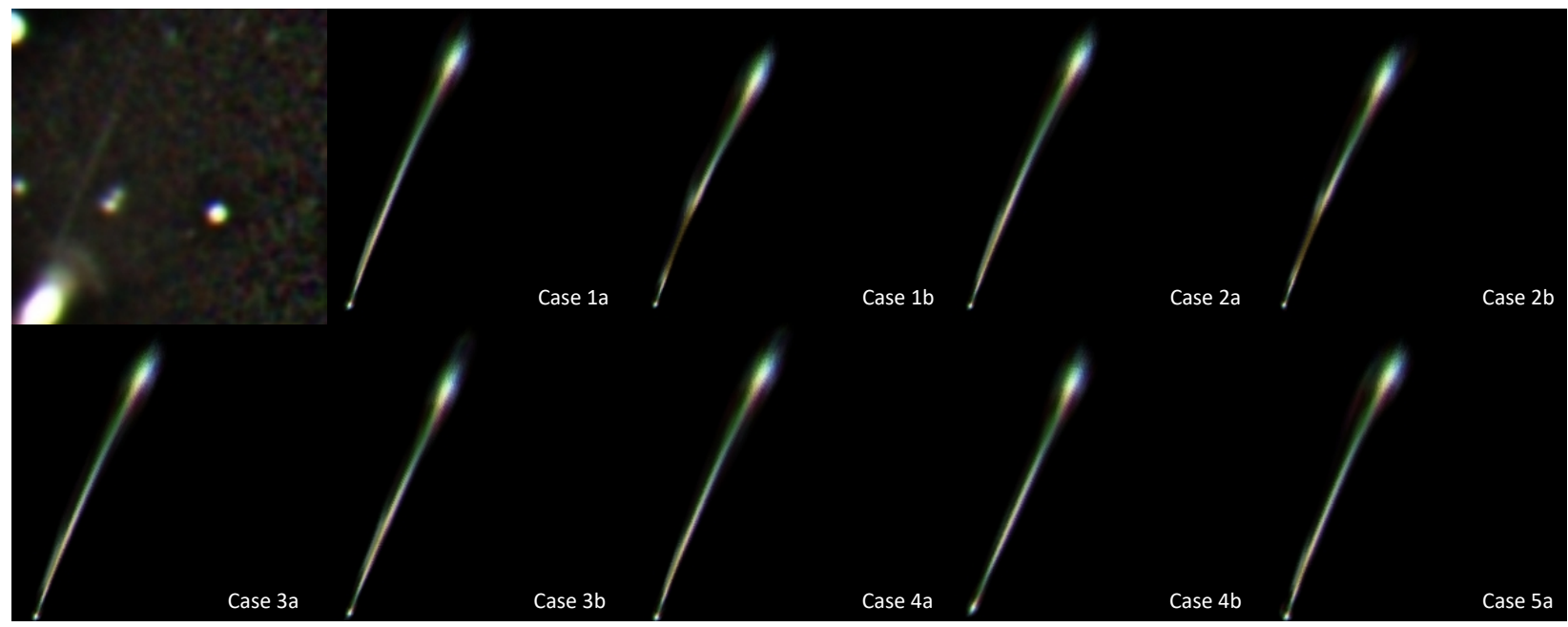

Fig. C.1. Original and predicted images of arc $4 \mathrm{~b}$ using the delensed counter-image $4 \mathrm{c}$ as a template of the source for the nine different models discussed at the end of Sect. 3. The field of view and centre of the images are the same in all panels. The image used was filtered to reduce the light contribution from member galaxies of the cluster.

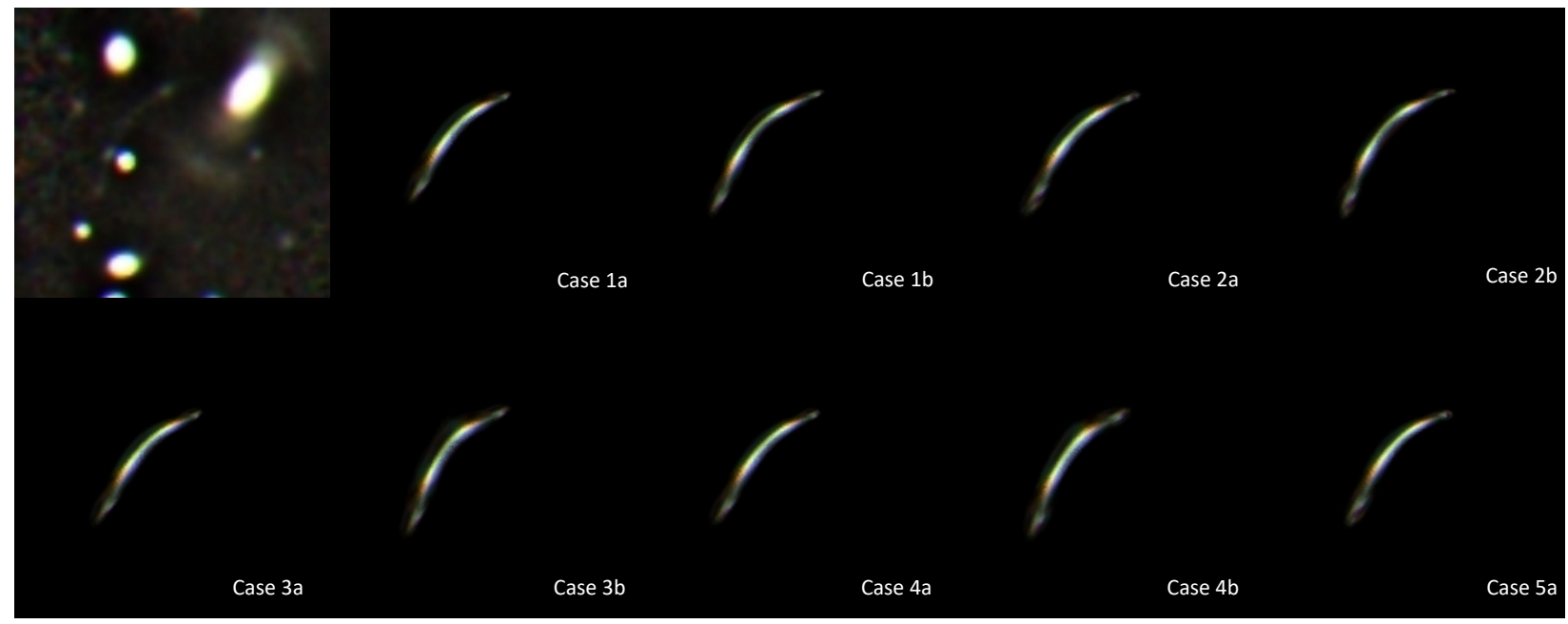

Fig. C.2. Original and predicted images of arc $7 \mathrm{c}$ using the delensed counter-image $7 \mathrm{a}$ as a template of the source for the nine different models discussed at the end of Sect. 3. The field of view and centre of the images are the same in all panels. The image used was filtered to reduce the light contribution from member galaxies of the cluster. 